\title{
Identification of microRNA-mRNA functional interactions in UVB-induced senescence of human diploid fibroblasts
}

Ruth Greussing ${ }^{1}$, Matthias Hackl ${ }^{2}$, Pornpimol Charoentong ${ }^{3}$, Alexander Pauck ${ }^{1}$, Rossella Monteforte ${ }^{2}$, Maria Cavinato ${ }^{1}$, Edith Hofer ${ }^{3}$, Marcel Scheideler ${ }^{4}$, Michael Neuhaus ${ }^{1}$, Lucia Micutkova' ${ }^{1}$ Christoph Mueck ${ }^{1}$, Zlatko Trajanoski ${ }^{3}$, Johannes Grillari, ${ }^{2,5}$ and Pidder Jansen-Dürr ${ }^{*}$

\begin{abstract}
Background: Cellular senescence can be induced by a variety of extrinsic stimuli, and sustained exposure to sunlight is a key factor in photoaging of the skin. Accordingly, irradiation of skin fibroblasts by UVB light triggers cellular senescence, which is thought to contribute to extrinsic skin aging, although molecular mechanisms are incompletely understood. Here, we addressed molecular mechanisms underlying UVB induced senescence of human diploid fibroblasts.

Results: We observed a parallel activation of the p53/p21 WAF1 and p16 1 NK4a/pRb pathways. Using genome-wide transcriptome analysis, we identified a transcriptional signature of UVB-induced senescence that was conserved in three independent strains of human diploid fibroblasts (HDF) from skin. In parallel, a comprehensive screen for microRNAs regulated during UVB-induced senescence was performed which identified five microRNAs that are significantly regulated during the process. Bioinformatic analysis of miRNA-mRNA networks was performed to identify new functional mRNA targets with high confidence for miR-15a, miR-20a, miR-20b, miR-93, and miR-101. Already known targets of these miRNAs were identified in each case, validating the approach. Several new targets were identified for all of these miRNAs, with the potential to provide new insight in the process of UVB-induced senescence at a genome-wide level. Subsequent analysis was focused on miR-101 and its putative target gene Ezh2. We confirmed that Ezh2 is regulated by miR-101 in human fibroblasts, and found that both overexpression of miR-101 and downregulation of Ezh2 independently induce senescence in the absence of UVB irradiation. However, the downregulation of miR-101 was not sufficient to block the phenotype of UVB-induced senescence, suggesting that other UVB-induced processes induce the senescence response in a pathway redundant with upregulation of miR-101.
\end{abstract}

Conclusion: We performed a comprehensive screen for UVB-regulated microRNAs in human diploid fibroblasts, and identified a network of miRNA-mRNA interactions mediating UVB-induced senescence. In addition, miR-101 and Ezh2 were identified as key players in UVB-induced senescence of HDF.

Keywords: Aging, Stress-induced senescence, miR-101, Ezh2, Epigenetics, UVB

\footnotetext{
* Correspondence: Pidder.Jansen-Duerr@uibk.ac.at

${ }^{1}$ Institute for Biomedical Aging Research, Austrian Academy of Sciences,

Rennweg 10, Innsbruck 6020, Austria

Full list of author information is available at the end of the article
} 


\section{Background}

Cellular senescence is controlled through a variety of regulatory mechanisms with particular contribution of the p53 [1] and Rb [2] tumor suppressor pathways. Briefly, p53 is activated in response to telomere damage or other kinds of DNA damage and orchestrates the transcriptional activation of a variety of downstream genes contributing to growth arrest, such as the CDK inhibitor $\mathrm{p} 21^{\mathrm{WAF} 1}$. In a parallel pathway, upregulation of the CDK inhibitor $\mathrm{p} 16^{\mathrm{INK} 4 \mathrm{a}}$ leads to the inhibition of phosphorylation of the retinoblastoma protein $(\mathrm{pRb})$, thereby enforcing prolonged cell cycle arrest [3], which is also a useful marker for senescence in human tissues [4]. Activation of $\mathrm{p} 16^{\mathrm{INK} 4 \mathrm{a}}$ triggers the appearance of senescence-associated heterochromatin foci [5], at least in some cell types. Activation of the p53/p21 ${ }^{\mathrm{WAF} 1}$ and $\mathrm{p} 16^{\mathrm{INK} 4 \mathrm{a}} / \mathrm{pRb}$ pathways represent two alternative scenarios for senescence initiation, and there is evidence that parallel activation of both pathways leads to enforced senescence [6], also referred to as "deep senescence" [7]. The relative importance of each of these pathways for cellular senescence seems to depend on the cell type. It should be noted that, besides these central pathways, a variety of signals have been shown to initiate a senescence response [8].

Stress-induced premature senescence (SIPS) has been identified as a model for telomere-independent senescence that can be initiated by various forms of stress, including oxidative stress, irradiation, replicative stress and oncogene activation [9]. In the case of skin derived human diploid fibroblasts (HDF), repeated mild treatment with UVB has been established as an experimental model for extrinsic skin aging [10], which depends on the accumulation of senescent cells, in particular fibroblasts in the dermis [9]. UVB irradiation is known to induce aspects of a DNA damage response, and it was reported that knocking down p53 alleviates but does not abrogate the senescence response of HDF to repeated UVB stress [11], suggesting that both p53-dependent and -independent pathways may cooperate to enforce UVB-induced senescence. However, UVB irradiation is known to affect a variety of intracellular signal transduction pathways $[12,13]$ and the precise sequence of events during establishment of UVB-induced premature senescence remained elusive.

Recent studies have emphasized an important role of non-coding RNAs, also referred to as microRNAs, as regulators of gene expression [14]. MicroRNAs are generated from larger precursor RNAs and were shown to interfere with the expression of protein coding genes by several mechanisms, including i) destabilization of specific mRNAs and ii) prevention of translation of specific mRNAs. Messenger RNAs targeted by microRNAs usually contain short sequences of homology [15]. It is known that changes in microRNA expression are contributing to cellular senescence [16] and organismic aging $[17,18]$; however, the role of microRNAs, if any, in UVB-induced senescence of human fibroblasts is only poorly understood. In the present communication, we have addressed molecular mechanisms underlying the establishment of premature senescence in human fibroblasts exposed to repeated series of mild UVB irradiations, as a model system to monitor molecular processes involved in extrinsic skin aging. We identified a small set of microRNAs, which are differentially regulated during the process and performed transcriptome analysis, in combination with advanced bioinformatics, to identify potential targets for these microRNAs.

\section{Results}

\section{UVB-induced changes in gene expression}

Human diploid fibroblasts (HDF, strain HFF-2) derived from newborn foreskin were subjected to eight consecutive UVB treatments of $4000 \mathrm{~J} / \mathrm{m}^{2}$ during four days. Under these conditions no overt cell death was observed (data not shown). Cell proliferation was strongly inhibited, and UVB-treated cells performed less than 2 population doublings (PDL) over 15 days of the experiment, whereas untreated cells underwent 12 PDL in the same time period. The growth arrest phenotype reached by repeated mild UVB stress resembled cellular senescence, as judged by the changes of cell morphology and the accumulation of a large percentage of cells that stained positive for senescence-associated $\beta$-galactosidase (SA- $\beta$ gal) (Additional file 1: Figure S1). As could be expected, UVB treatment led to a strong but transient phosphorylation of p53 on serine 15 , most notable at day 4, i.e. after application of the last stress, indicative of p53 activation, consistent with previous observations [19]. The overall levels of p53 protein were also increased, in line with previous findings [20]. Activation of p53 also resulted in the upregulation of its downstream effector p21 WAF1, which is known to enforce cellular senescence in response to DNA damage [21]. We also addressed effects of UVB treatment on the $\mathrm{p} 16^{\mathrm{INK} 4 \mathrm{~A}} / \mathrm{pRb}$ pathway, representing the other important pathway relevant for cellular senescence [22]. As could be expected from the observed upregulation of $\mathrm{p} 21^{\mathrm{WAF} 1}$, UVB treatment led to the rapid disappearance of phosphorylated species of the retinoblastoma protein ( $\mathrm{pRb}$ ), which was accompanied by a delayed but significant upregulation of the CDK inhibitor $\mathrm{p} 16^{\mathrm{INK} 4 \mathrm{~A}}$ (Additional file 1: Figure S1). Together the data suggest that mild UVB stress induces premature cellular senescence and that both the p53/p21 WAF1 axis and the $\mathrm{p} 16^{\mathrm{INK} 4 \mathrm{~A}} / \mathrm{pRb}$ pathway are involved in the senescence response, consistent with previous reports [23-25]. The same protocol of repeated mild UVB stress induced premature senescence also in two other strains 
of human diploid fibroblasts, HFF-1 and PFF, with a very similar kinetics (Additional file 2: Figure S2), along with the activation of the $\mathrm{p} 53 / \mathrm{p} 21^{\mathrm{WAF} 1}$ and $\mathrm{pRb} / \mathrm{p} 16^{\mathrm{INK} 4 \mathrm{~A}}$ pathways (Additional file 3: Figure S3).

To characterize the response of HDF to UVB-induced premature senescence, genome-wide transcriptome analysis was performed at various time points. Applying a cutoff of $>1.5$ fold regulation, this analysis revealed the upregulation of 1219 genes and the downregulation of another 1077 genes in response to mild UVB treatment. Changes in gene expression level were most pronounced at days 7 and 9 after beginning of the treatment. Initial analysis of UVB-responsive pathways by Ingenuity ${ }^{\text {Tm }}$ Software, based on the results of Affymetrix chip analyses, revealed several distinct molecular pathways preferentially affected by the treatment, including G1/S cell cycle checkpoint, DNA damage checkpoint, p53 signaling pathway, cell migration, aryl hydrocarbon receptor signaling, pyrimidine metabolism and nicotinate and nicotinamide metabolism (Figure 1A). For day 9 after UVB irradiation, three independent Affymetrix chip experiments were performed, which revealed significant upregulation $(\mathrm{p}<0.05)$ for a total of 632 genes, whereas 716 genes were significantly downregulated $(\mathrm{p}<0.05)$. The list of all regulated genes and corresponding Affymetrix expression data are provided as Additional file 4: Table S1. From these genes, a set of 67 genes was selected for an independent analysis by RT-qPCR, which confirmed all regulations revealed by Affymetrix chips (Figure 1B).

\section{microRNAs regulated in UVB-induced senescence}

We also determined the expression levels of $806 \mathrm{miR}$ NAs in UVB treated compared to control cells. The relative expression levels of miRNAs at various time points after UVB stress, as determined by miRNA array, are shown in Additional file 5: Table S2. We used publicly available and in-house developed tools, to compare expression profiles of all miRNAs and the 67 validated mRNAs (Figure 1B), and predicted target pairs to score and identify high confidence miRNA targets. Thereby, eight miRNAs (miR-15a, miR-17, miR-20a, miR-20b, miR-34, miR-93, miR-101, miR-155) were identified for which regulated mRNA targets were found with high confidence. Subsequently, the expression levels of selected miRNAs were analyzed by qPCR. In these experiments, data obtained by the miRNA array for miR-15a, miR-20a, miR-20b, miR-93, and miR-101 were confirmed (Figure 2); whereas miR-17, miR-34 and miR-155 were also regulated in UVB-treated cells in accordance with the miRNA array results, the observed differences did not reach statistical significance (data not shown). Expression levels for miR-15a, miR-20a, miR-20b, miR-93, and miR101 are shown in Figure 3, along with their established target mRNAs.

\section{miRNA-mRNA regulatory networks in UVB-induced senescence}

High confidence targets were identified for miR-20a and miR-20b encoded by the miR-17-92 cluster which is known to synergize with Myc in cancer development [26], probably through repression of p21 ${ }^{\mathrm{WAF} 1}$ expression at the post-transcriptional level [27]. However, there is also evidence that miR-17-92 blocks E2F-dependent steps in the regulation of angiogenesis [28]. Our analysis confirmed a high confidence interaction between both miR-20a and miR-20b with p21 ${ }^{\text {WAF1 }}$ (CDKN1A), p15 $5^{\mathrm{INK} 4 \mathrm{~B}}$ (CDKN2B), RUNX1, and vEGF-A (Figure 4A,B), thereby validating the analytical procedure. E2F1 and Cyclin D1 are predicted targets for both miR-20a and miR-20b. Whereas decreased expression of miR-20a/b was not correlated with altered mRNA levels of E2F1 and Cyclin D1 (Figure 4A,B), upregulation of Cyclin D1 gene expression during UVB-induced senescence was observed at the protein level (Figure $4 \mathrm{C}$ ). Results of the bioinformatic analysis suggested several so far unreported potential targets for miR-20a and miR-20b in the context of UVB-induced senescence. Thus, DRAM, IDS, NFAT5, EGR2, CCND2, and RARB were identified as potential high confidence interactions for both miR-20a and miR20b (Figure 4A,B). In addition, the data suggest TIMP3, ETV1, B2M, IGFBP-3, and RRAS2 as potential targets for miR-20a (Figure 4A), and TGM2, CPE, RHOJ, and SERPING1 as potential targets for miR-20b (Figure 4B).

miR-93 is known to inhibit angiogenesis by suppressing VEGF release [29], and contributes to silencing of $\mathrm{p} 21^{\mathrm{WAF} 1}$ gene expression after DNA damage [30]. Moreover, miR-93 increases survival in cisplatin-resistant ovarian cancer cells, by directly targeting PTEN and upregulation of the AKT signaling pathway [31]. Our analysis in UVB-induced senescence confirmed a high confidence interaction of miR-93 with CDKN1A and vEGF-A (Figure 5A), thereby validating the analytical procedure. Results of the bioinformatic analysis suggested DRAM, PIK3IP1, DKK2, Serpin G1, ADAMTS5, TIMP3, BTG2, RUNX1 and EGR2 as potential targets for miR-93 (Figure 5A).

miR-15a, along with miR-16, is commonly deleted in human chronic lymphocytic leukemia [32] and known to target multiple oncogenes, including BCL2, MCL1, CCND1, and WNT3A [33]. Our analysis confirmed a high confidence interaction between miR-15a and vEGF-A in UVB-induced senescence (Figure 5B), thereby validating the analytical procedure. Results of the bioinformatic analysis suggested PAPPA, APOD, RRAS2, Runx1, RARB, BTG2, Notch3 and SFRP1 as potential targets for miR-15 (Figure 5B).

miR-101 is known to suppress expression of the histone methyltransferase Ezh2 [34,35]. Our analysis confirmed a high confidence interaction between miR-101 


\section{A} Representative pathways

\section{B}

\begin{tabular}{|l|c|c|}
\hline & $\begin{array}{c}\text { Up. } \\
\text { regulated }\end{array}$ & $\begin{array}{c}\text { Down- } \\
\text { regulated }\end{array}$ \\
\hline $\begin{array}{l}\text { Cell Cycle: G2/M DNA Damage } \\
\text { Checkpoint Regulation }\end{array}$ & 1 & 10 \\
\hline $\begin{array}{l}\text { Role of BRCA1 in DNA Damage } \\
\text { Response }\end{array}$ & 2 & 18 \\
\hline $\begin{array}{l}\text { Cell Cycle: G1/S Checkpoint } \\
\text { Regulation }\end{array}$ & 4 & 8 \\
\hline p53 Signalling & 8 & 5 \\
\hline $\begin{array}{l}\text { Aryl Hydrocarbon Receptor } \\
\text { Signalling }\end{array}$ & 7 & 11 \\
\hline Pyrimidine Metabolism & 4 & 16 \\
\hline $\begin{array}{l}\text { Nicotinate and Nicotinamide } \\
\text { Metabolism }\end{array}$ & 3 & 6 \\
\hline $\begin{array}{l}\text { Hepatic Fibrosis / Hepatic Stellate } \\
\text { Cell Activation }\end{array}$ & 12 & 1 \\
\hline
\end{tabular}

\section{Microarray expression}
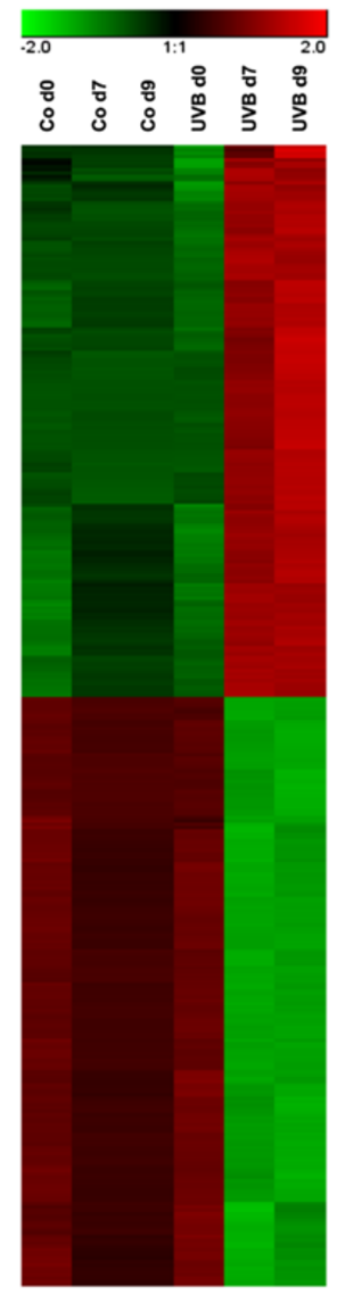

qPCR expression
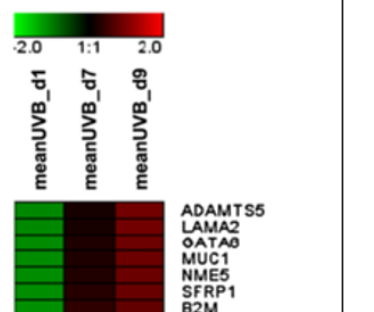

Figure 1 Transcriptome analysis of UVB-induced senescence.

A. Ingenuity pathway analysis. Activated pathways were determined by Ingenuity System Pathway Analysis software (http://www.ingenuity. $\mathrm{com} / \mathrm{b}$ by Core Analysis. Shown are eight significantly activated pathways (threshold $\mathrm{p}$-value $\leq 0.05$ ). B. In silico analysis of mRNA expression change in response to UVB irradiation. (left) The differential gene expression profile from total RNA was examined by Affymetrix GeneChip analysis (cutoff of $>1.5$ fold regulation) 1, 7, and 9 days between control and UVB irradiated of HDF samples. The expression values were sorted by level of Ezh2 expression (rows). (right) Heatmap of selected 67 genes were determined by RT-PCR and sorted by level of Ezh2 expression (rows), red representing overexpression and green representing underexpression of the transcript.

and Ezh2 in UVB-induced senescence (Figure 6A), thereby validating the analytical procedure. Accordingly, Ezh2 expression was significantly downregulated at both mRNA and protein level in UVB treated cells (Figure 6B). Results of the bioinformatic analysis suggested BIRC5, NAT13, and CXCL12 as potential targets for miR-101. (Figure 6A). Whereas the majority of analyzed potential mRNA targets for miR-101 displayed a positive correlation with miR-101 levels (red lines in Figure 6A), the biological meaning of these interactions remains to be established.

To further validate the bioinformatics-based target selection, regulation of several newly identified candidate genes by specific microRNAs was addressed in HDF overexpressing miR-15a, miR-20a, and miR-93, respectively. In all cases, microRNA levels were significantly ( $>20$ fold) increased by transfection (data not shown). For the genes RARB, RUNX1 and CDKN2B, we found that overexpression of the appropriate microRNA species (miR-15a and miR-20a, respectively) reduced protein levels of the respective gene products (Additional file 6: Figure S4). Whereas these findings do not prove binding of microRNAs $15 \mathrm{a}$ and $20 \mathrm{a}$ to the 3 -UTR of the target genes, downregulation of protein expression by overexpression of selected miRNAs provides a functional validation of the bioinformatics approach. The mRNAs for several other genes, including VEGFA, RHOJ, and NOTCH3 were significantly down-regulated by the appropriate microRNAs (data not shown); however, the limited availability of high-quality antibodies precluded determination of protein expression levels in these cases.

\section{A role of miR-101/Ezh2 in UVB-induced senescence?}

To address the functionality of the miR-101-Ezh2 interaction in UVB-induced senescence of HDF and their importance for UVB-induced senescence, we addressed the potential of these molecules to affect cellular senescence in HDF. In a first set of experiments, we analyzed consequences of overexpression of miR-101. Using reverse transfection, miR-101 was overexpressed in human diploid fibroblasts, which resulted in a clear upregulation of 

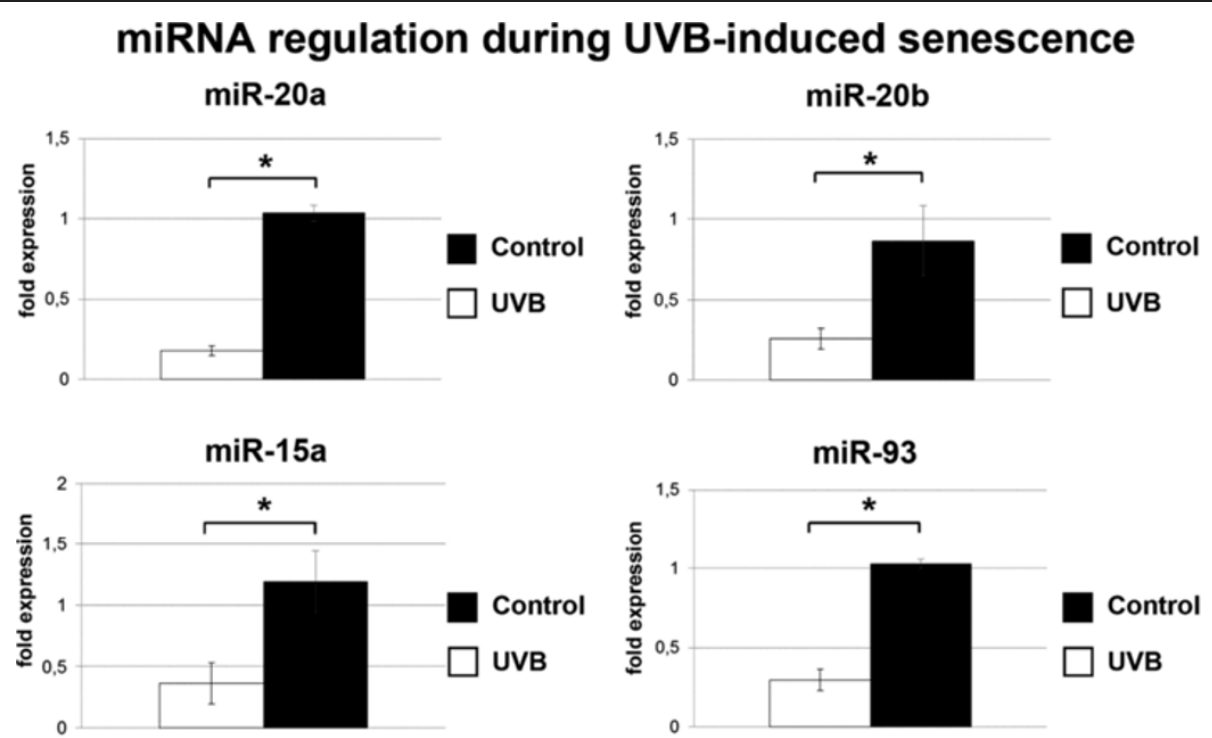

miR-101

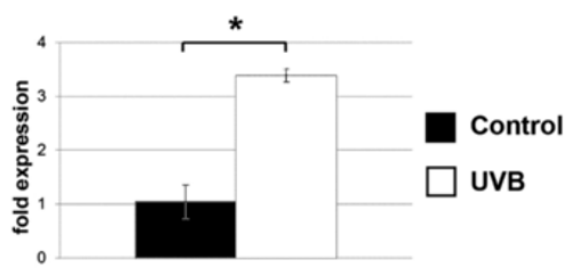

Figure 2 Validated miRNA expression levels. miRNA was isolated from UVB treated and control cells. miRNA expression levels for miR-20a, miR-20b, miR-15a, and miR-93 were determined by Locked nucleic acid (LNA)-miRNA microarray. Experiments were performed in triplicates. ${ }^{*} p<0.01 ;{ }^{* *} p<0.001$

miR-101 (Figure 7A). The strong upregulation of miR101 induced a significant downregulation in the level of Ezh2 mRNA (Figure 7B) and protein (Figure 7C). In consequence, overexpression of miR-101 was sufficient to reduce the rate of proliferation of human diploid fibroblasts (Figure 7D) and induced a significant increase in the number of SA- $\beta$-gal positive cells (Figure $7 \mathrm{E}$ ). These experiments suggest that indeed miR-101 has the potential to downregulate Ezh2 mRNA and protein levels in HDF, and this can lead to growth arrest and entry into premature senescence. To knock down Ezh2 expression in HDF, lentiviral vectors carrying Ezh2targeting shRNAs were used. Out of five shRNAs tested, \#73 and \#75 effected a significant downregulation of Ezh2 levels in transfected cells (Additional file 7: Figure S5). Lentiviral vectors were prepared carrying these shRNAs and used to infect HDF. Ezh2 knockdown led to a downregulation of cell proliferation, along with a significant increase in the percentage of SA- $\beta$-gal positive cells, suggesting that knocking down Ezh2 can

Representative miRNA expression

\begin{tabular}{|c|c|c|c|c|l|}
\hline \multicolumn{5}{|c|}{ miRNA expression (fold expression) } & \\
\hline miRNA & $\mathbf{d} 1$ & $\mathbf{d} 4$ & $\mathrm{~d} 7$ & $\mathrm{~d} 9$ & \multicolumn{1}{|c|}{ target mRNA } \\
\hline hsa-miR-20a & $-1,067$ & $-1,987$ & $-1,598$ & $-2,629$ & VEGFA, E2F1, RUNX1, CCND1 \\
\hline hsa-miR-20b & $-1,046$ & $-1,702$ & $-1,288$ & $-2,073$ & CDKN1A, VEGFA, E2F1, CCND1, RUNX1 \\
\hline hsa-miR-15a & 1,037 & $-1,661$ & $-1,666$ & $-2,274$ & BCL2, VEGFA \\
\hline hsa-miR-93 & $-1,034$ & $-1,567$ & $-1,613$ & $-2,722$ & E2F1, VEGFA, CDKN1A \\
\hline hsa-miR-101 & 1,024 & $-1,212$ & 2,465 & 1,244 & MYCN, EZH2 \\
\hline
\end{tabular}

Figure 3 microRNA expression level. The expression levels of 806 miRNAs were determined at day 1, 4, 7 and 9 by LNA microarray. Displayed are five selected miRNAs with the corresponding mRNA targets, identified by DIANA LAB (http://diana.cslab.ece.ntua.gr/tarbase/) and miRecords (http://mirecords.biolead.org/). In red are miRNAs and target mRNAs that are upregulated, downregulated genes are colored in blue and not regulated genes are in black. 


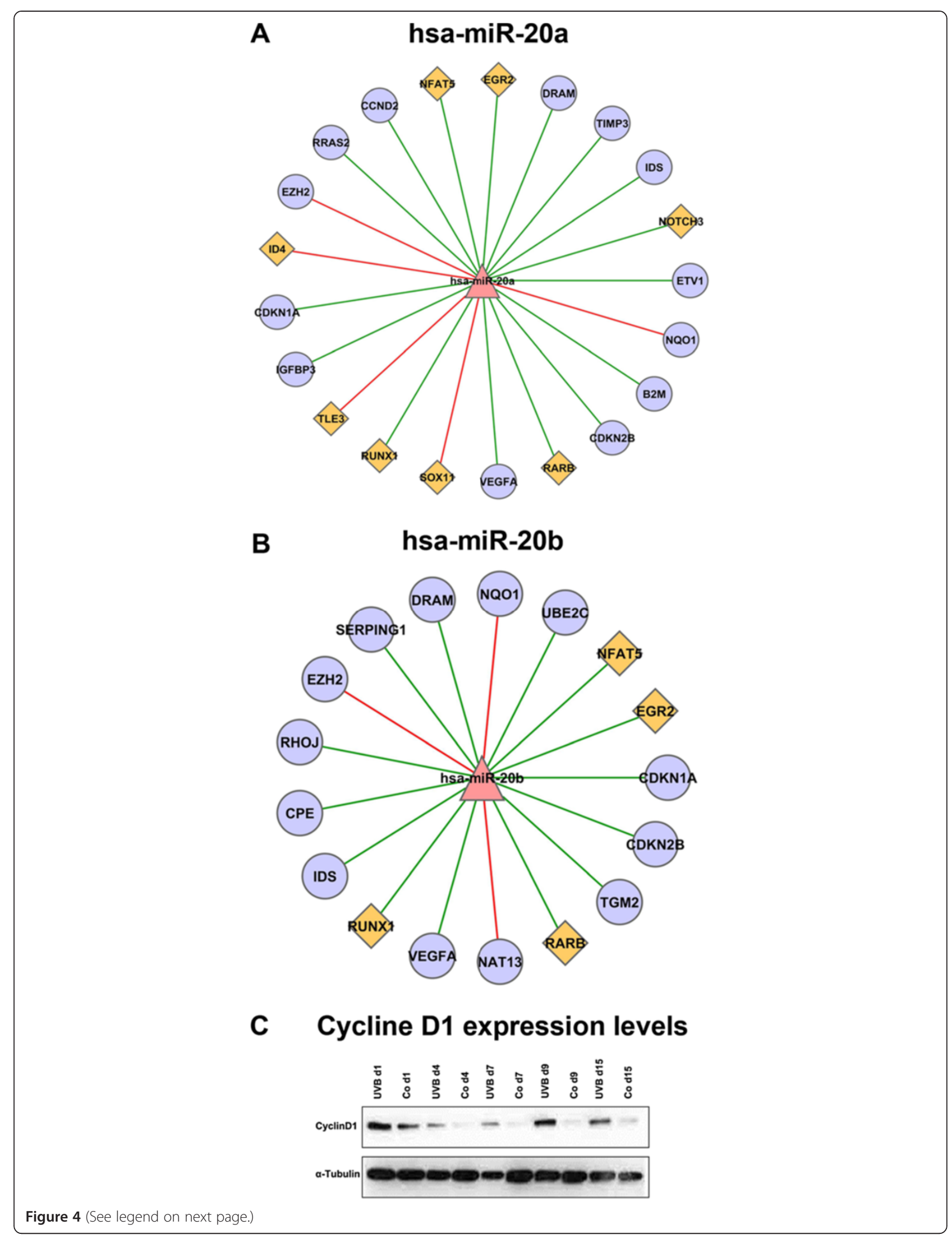




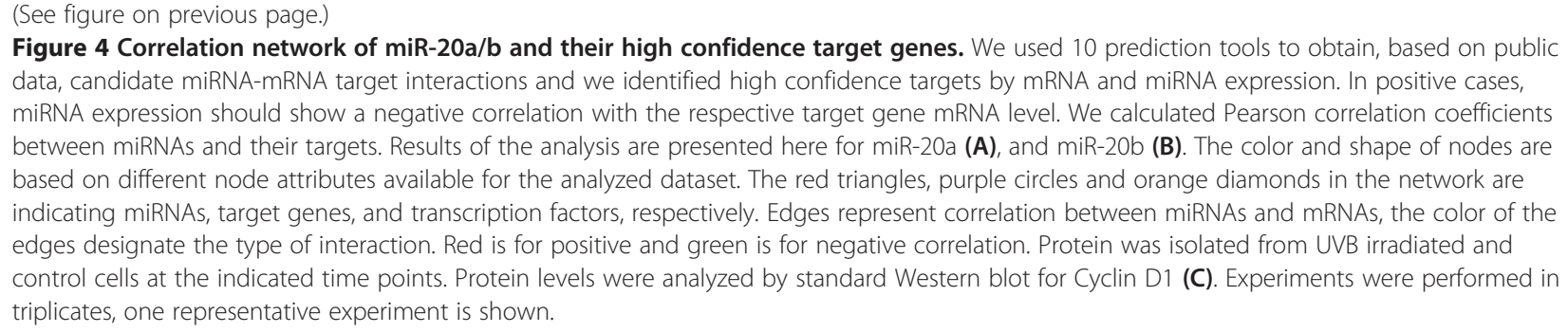

induce premature senescence of HDF (Additional file 7: Figure S5).

To address the role of miR-101 in UVB-induced cellular senescence, we attempted to experimentally reduce miR-101 levels by transfection of miR-101 inhibitory RNAs. This treatment was started one day before UVB treatment and continued thereafter, in order to keep miR-101 levels constantly low. Using reverse transfection, the levels of miR-101 were significantly reduced in control HDF (Figure 8A) and miR-101 knockdown efficiently abrogated miR-101 upregulation in UVB-treated fibroblasts (Figure 8B). However, Ezh2 mRNA was not significantly upregulated in miR-101 depleted cells, irrespective of UVB treatment (Figure 8A,B); and miR-101 knockdown failed to increase Ezh2 protein levels in both cases (data not shown). To address potential mechanisms underlying the failure to upregulate Ezh2, Ezh2 was also overexpressed by lentiviral vectors. Both in HDF and in easy-to-transfect human osteosarcoma (U2-OS) cells, overexpression of Ezh2 mRNA was transient and no elevation of Ezh2 protein levels was observed in HDF (Additional file 8: Figure S6), for reasons that remain to be established. Together, these experiments indicate that reduction of miR-101 was not sufficient to rescue Ezh2 expression in UVB-treated cells. Accordingly, the phenotype of UVB-induced cellular senescence was not significantly affected by preventing the UVB-induced upregulation of miR-101 (Figure 8C). Consistent with this observation, knockdown of miR101 also failed to significantly reduce the percentage of SA- $\beta$-gal positive cells after UVB treatment (data not shown).

\section{Discussion}

In this communication, we addressed molecular mechanisms underlying UVB-induced senescence of human diploid fibroblasts. Using genome-wide transcriptome analysis, we identified a transcriptional signature of UVB-induced senescence. In parallel, a comprehensive screen for microRNAs regulated during UVB-induced senescence was performed and five microRNAs were identified that are significantly regulated during the process. Subsequent analysis revealed several well established miRNA-mRNA regulatory interactions including
miR-101/Ezh2, thereby validating the assay. In addition, several new miRNA-mRNA regulatory interactions were identified to occur in UVB-induced senescence. Overexpression of miR-101 and downregulation of Ezh2 independently induced senescence in the absence of UVB irradiation. However, the downregulation of miR-101 was not sufficient to block the phenotype of UVB-induced senescence, suggesting that other UVB-induced processes contribute to the senescence response, partially redundant with upregulation of miR-101. These findings extend our knowledge of miR-mRNA regulatory interactions, and at the same time provide a rich resource for data mining to identify new players in UVB-induced senescence and potential targets for interventions in extrinsic skin aging.

\section{Transcriptional profiling of UVB-induced premature senescence}

Whereas a low-density DNA array was used previously to study changes in the level of 240 senescence-related genes in UVB-induced senescence of HDF [11], the current study provides the first genome-wide transcriptional analysis of UVB-induced senescence. As could be expected [1], activation of p53 signaling and suppression of the G1/S transition were observed along with a pronounced DNA damage response, as revealed by pathway analysis tools. We also observed concerted regulation of genes in the aryl hydrocarbon receptor signaling and hepatic fibrosis/heaptic stellate activation pathways, which conceivably reflect specific responses to UVB irradiation, used here to trigger the senescence response. Of note, there were also distinct changes in the regulation of genes coding for enzymes in the pyrimidine and nicotinamide metabolism; however, more work will be required to fully understand the implications of these alterations for UVBinduced senescence. Conceivably, the transcriptional signature of UVB-induced senescence, as shown here, will pinpoint new targets for intervention in extrinsic photoaging, which will be investigated in follow-up studies.

\section{miRNA-mRNA regulatory networks in UVB-induced senescence}

miR-34, a known transcriptional target of p53 [36], was strongly upregulated at day 7 , as expected from the observed activation of p53. Similarly, downregulation of 


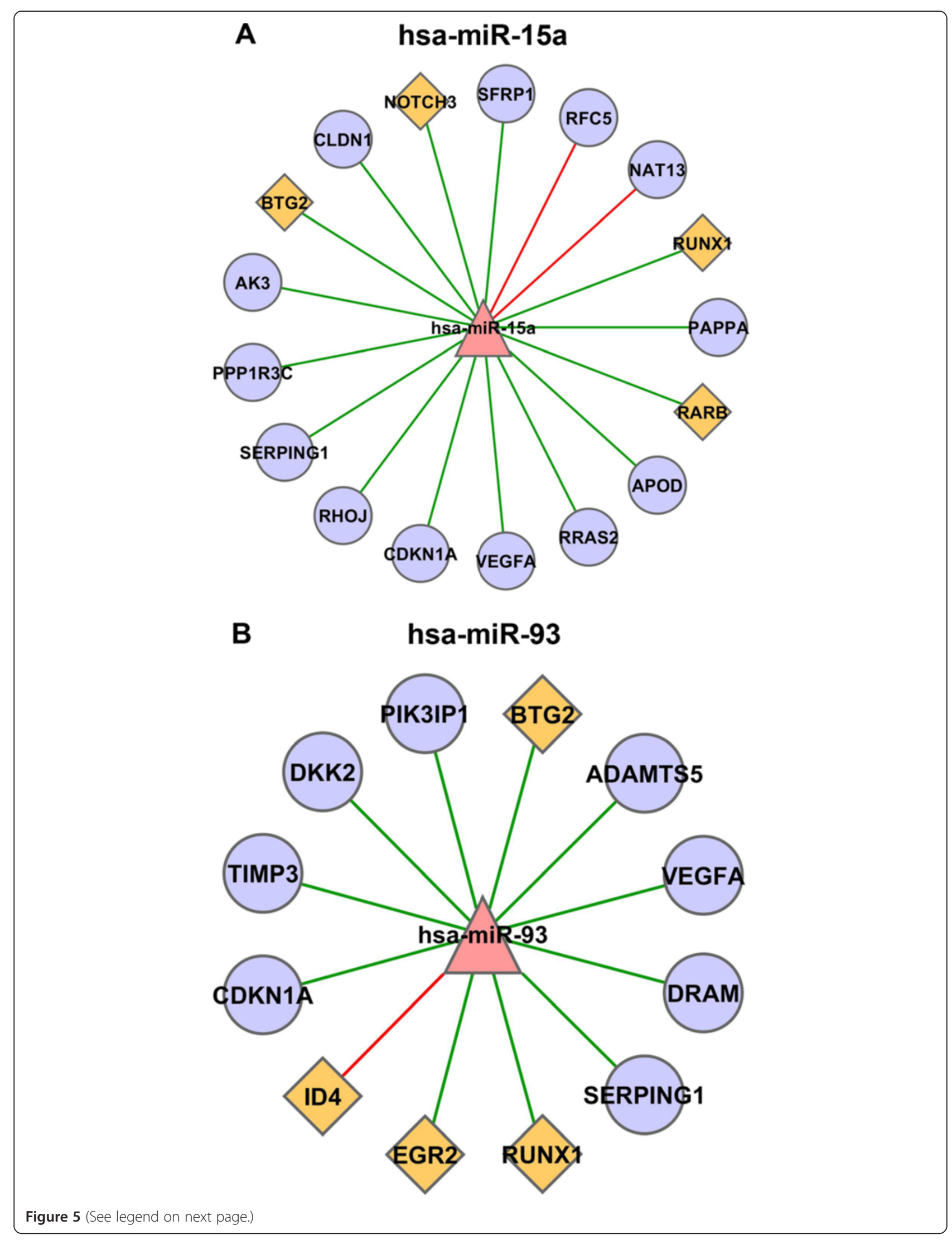


(See figure on previous page.)

Figure $\mathbf{5}$ Correlation networks of miR-93/miR-15 and their high confidence target genes. We used 10 prediction tools to obtain, based on public data, candidate miRNA-mRNA target interactions and we identified high confidence targets by mRNA and miRNA expression. We calculated Pearson correlation coefficients between miRNAs and their targets. Results of the analysis are presented here for miR-93 (A), and miR-15 (B). The color and shape of nodes are based on different node attributes available for the analyzed dataset. The red triangles, purple circles and orange diamonds in the network are indicating miRNAs, target genes, and transcription factors, respectively. Edges represent correlation between miRNAs and mRNAs, the color of the edges designate the type of interaction. Red is for positive and green is for negative correlation.

miR-20a and miR-20b is consistent with previous observations suggesting that members of the miR-17-92 cluster are commonly downregulated in various senescence models as well as in organismal aging [16]. These observations validated the miRNA screening approach. It is known that miRNAs regulate both stability and translation of mRNAs, and in most cases upregulation of miRNAs leads to the inhibition of gene function [37]. The miR-17-92 cluster, containing the microRNA-17-18-1920-92 polycistron, cooperates with Myc in tumorigenesis [26], probably by silencing of p $21^{\text {WAF1 }}$ expression at the post-transcriptional level [27]. On the other hand, miR17-92 inhibited proliferation and metastasis of pancreatic carcinoma cells [38], blocked E2F-dependent steps in the regulation of angiogenesis [28], and repressed endothelial cell migration [39], suggesting cell type specific effects. Our finding that E2F1 and VEGFA are relevant targets for $\mathrm{miR}-20 \mathrm{a} / \mathrm{b}$ in the context of UVB-induced senescence is consistent with these data.

Although frequently overexpressed in human malignancies, miR-93 may actually function as a tumor suppressor gene. Accordingly, miR-93 abrogated VEGF protein secretion, suggesting that miR-93 interferes with angiogenesis [29], blocked tumor development in mammary fat pads [40], and suppressed proliferation of human colon cancer stem cells [41]. However, miR-93 was also shown to i) promote tumor growth and angiogenesis by targeting integrin- $\beta 8$ [42], ii) to contribute to silencing of $\mathrm{p} 21^{\mathrm{WAF} 1}$ gene expression after DNA damage [30], and iii) to promote cell proliferation and clonogenicity of HepG2 Cells [43], suggesting that effects of miR93 depend on cellular context. Upregulation of p21 WAF1 gene expression in UVB-treated cells was correlated with the downregulation of miRNAs $20 \mathrm{a} / \mathrm{b}$ and 93 , known to target p21 WAF1 $[27,30,44-46]$, and the identification of E2F1 and VEGFA as relevant targets for miR-93 in the context of UVB-induced senescence is consistent with the available data.

miR-15a, along with miR-16, was the first microRNA linked to cancer because both genes are commonly deleted in human chronic lymphocytic leukemia [32]. Expression of miRNAs encoded by the miR-15/16 cluster inhibits cell proliferation, promotes apoptosis of cancer cells, and suppresses tumorigenicity both in vitro and in vivo. miR-15a and miR-16-1 function by targeting multiple oncogenes, including BCL2, MCL1, CCND1, and WNT3A [33,47]. In contrast to their function as tumor suppressors, miR-15 can also promote tumor growth and progression, when expressed in cancerassociated fibroblasts [48]. The identification of VEGFA as relevant target for miR-15 in the context of UVBinduced senescence is consistent with these data. On the other hand, mRNA levels of E2F1 and $\mathrm{Bcl} 2$ were downregulated in UVB-treated cells (Additional file 4: Table S1 and data not shown), although three miRNAs known to target E2F1 (miR 20a, 20b and 93) and one miRNA known to target $\mathrm{Bcl} 2$ (miR-15a) were all significantly downregulated in UVB-treated cells (Figure 3). It is conceivable that, in these cases, additional regulatory processes take place, which remain to be identified. A particular complication in the interpretation of the current results lies in pleiotropic effects of UVB irradiation, which are known to affect the expression of selected miRNAs and mRNAs (also shown here) but also may influence protein translation [49] and/or protein stability [50]. Accordingly, the assignment of miRNAs and gene expression changes reflects plausibility rather than a strict mechanistic dependence. Bioinformatics analysis and preliminary validation analysis performed in the current study highlighted several miRNA targets as potential modulators of UVB-induced fibroblast senescence that were not previously described, including the cdk inhibitor CDKN2B and the transcription factors RUNX1 and RARB. Whereas these findings establish CDKN2B, RUNX1 and RARB as functional target genes for miR-15a and miR$20 \mathrm{~A}$, respectively, the binding of these microRNAs to the 3'-UTR of the target genes remains to be confirmed by additional experiments.

\section{Regulation of UVB-induced senescence: the role of miR-101 and Ezh2}

The here reported data demonstrate for the first time the implication of miR-101/Ezh2 signaling in UVB-induced senescence of human dermal fibroblasts. In control experiments, upregulation of miR-101 and the concomitant downregulation of Ezh2 was also observed in two independent HDF strains, HFF-1 and PFF (Additional file 3: Figure S3), indicating that the regulation of these components is a conserved feature in the senescence response of human dermal fibroblasts to UVB irradiation. The inverse relationship between miR-101 and Ezh2 expression levels was noticed before [51,52] and Ezh2 has been shown by 


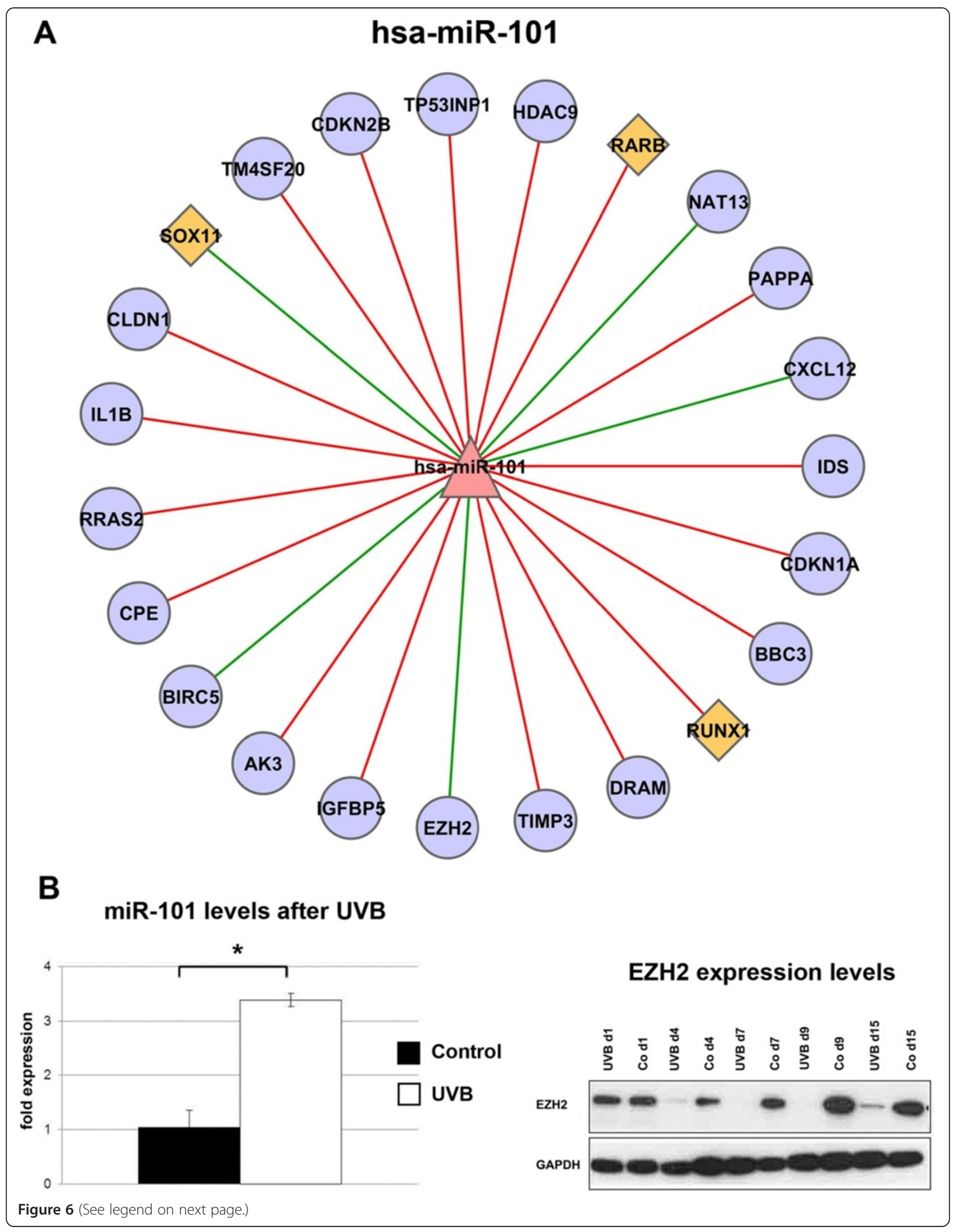


(See figure on previous page.)

Figure 6 Correlation network of miR-101 and its high confidence target genes. (A) We used 10 prediction tools to obtain, based on public data, candidate miRNA-mRNA target interactions and we identified high confidence targets by mRNA and miRNA expression. We calculated Pearson correlation coefficients between miRNAs and their targets. Results of the analysis are presented here for miR-101. The color and shape of nodes are based on different node attributes available for the analyzed dataset. The red triangles, purple circles and orange diamonds in the network are indicating miRNAs, target genes, and transcription factors, respectively. Edges represent correlation between miRNAs and mRNAs, the color of the edges designate the type of interaction. Red is for positive and green is for negative correlation. (B) Protein samples for the indicated time points were collected as described (left panel). Protein levels were determined for Ezh2 by standard Western blot analysis. Experiments were performed in triplicates, shown here is a representative result. miR-101 levels were determined by real-time qPCR as described (right panel). Data represents the mean \pm SE for three independent experiments. Co: untreated controls. ${ }^{*} p<0.01$; ${ }^{* *} p<0.001$.

others to play a role in cellular senescence [53,54]. When this communication was under revision, it was reported by others that miR-101 controlled Ezh2 function in cellular senescence of mouse embryonic fibroblasts [55]. In the present communication, functional interactions between miR-101 and Ezh2 in UVB-induced senescence of HDF were analyzed in more detail. For example, we tried by knockdown of miR-101 to rescue the expression levels of Ezh2 in UVB irradiated cells. Although knockdown of miR-101 was very efficient also in UVB treated cells, no corresponding increase in the level of Ezh2 mRNA or protein was observed, suggesting that expression of the Ezh2 gene is regulated by additional signals, which remain to be established. It is conceivable that p53 is responsible for the effect, since p53 was shown to suppress the Ezh2 gene promoter [56,57]. Conversely, we also tried to rescue the phenotype of UVB-induced senescence by overexpression of Ezh2 from a lentiviral overexpression vector. Whereas Ezh2 mRNA was significantly upregulated in infected cells, this did not lead to any detectable increase of Ezh2 protein levels, suggesting that overexpression of Ezh2 protein is not well tolerated, at least in HDF. The mechanisms underlying downregulation of Ezh2 in this cell type remain to be understood. After UVB irradiation, Ezh2 protein levels were rapidly reduced, even prior to the induction of miR-101 (Figure 3), strongly suggesting that several different UVB-dependent pathways converge to downregulate Ezh2, the upregulation of miR-101 being just one of several triggers for this process.

\section{Conclusions}

We report here a comprehensive screen for microRNAs and mRNAs regulated during UVB-induced senescence in human diploid fibroblasts. Using advanced bioinformatics solutions, we identified a network of miRNAmRNA interactions mediating UVB-induced senescence in this cell type, providing a rich resource for future data mining. The data reported in this communication illustrate the regulation of five distinct miRNAs during UVB-induced cellular senescence. Together the results obtained in this study suggest important roles for microRNAs miR-15, miR-20a/b, miR-93 and miR-101, and their mRNA targets, during UVB-induced senescence of human diploid fibroblasts.

\section{Methods}

\section{Chemicals}

All chemicals were purchased from Sigma, unless indicated otherwise.

\section{Cell culture}

Human diploid foreskin fibroblasts (HDF) were either purchased from ATCC (Manassas, VA) (HFF-1 \#SCRC-1041; HFF-2 \#SCRC-1042) or isolated from newborn foreskin (PFF) in our laboratory, as described [58]. Cells were used at passage 6 (HFF-2, PFF) and passage 10 (HFF-1). HDFs and human osteosarcoma cells (U2-OS; obtained from ATCC, Manassas, VA) were cultured in the same way in DMEM (Sigma) as described [59]. The cumulative population doublings (cPDL) were calculated using the following equation: $\mathrm{cPDL}=(\log (\mathrm{A})-\log (\mathrm{B})) / 0.301$ (A: number of cells at the end of one passage; $\mathrm{B}$ : number of cells that were seeded at the beginning of one passage).

\section{SA- $\beta$-galactosidase staining}

Senescence-associated- $\beta$-galactosidase (SA- $\beta$-gal) staining was used to determine the senescent status of the cells. To stain for SA- $\beta$-gal, cells were grown on 6-well plates and washed three times with PBS. Afterwards, the cells were fixed with $2 \%$ formaldehyde and $0.4 \%$ glutaraldehyde in PBS for 5 minutes at room temperature. Cells were washed three times with PBS and prepared for staining as described previously [59]. Therefore, cells were covered with staining solution $(150 \mathrm{mM} \mathrm{NaCl}$, $2 \mathrm{mM} \mathrm{MgCl}, 5 \mathrm{mM}$ potassium ferricyanide, $5 \mathrm{mM}$ potassium ferrocyanide, $40 \mathrm{mM}$ citric acid, $12 \mathrm{mM}$ sodium phosphate, $\mathrm{pH}$ 6.0, adding $1 \mathrm{mg} / \mathrm{mL}$ 5-bromo-4-chloro3-indolyl-b-D-galactoside [X-gal] directly before use) and incubated for $24 \mathrm{~h}$ at $37^{\circ} \mathrm{C}$ without light exposition. The reaction was stopped by washing off the staining solution with PBS. Cells were covered with PBS and blue staining indicating the presence of SA- $\beta$-gal can be detected under the microscope. To calculate the percentage of SA- $\beta$-gal positive cells, stained cells were counted and related to the total cell number. 


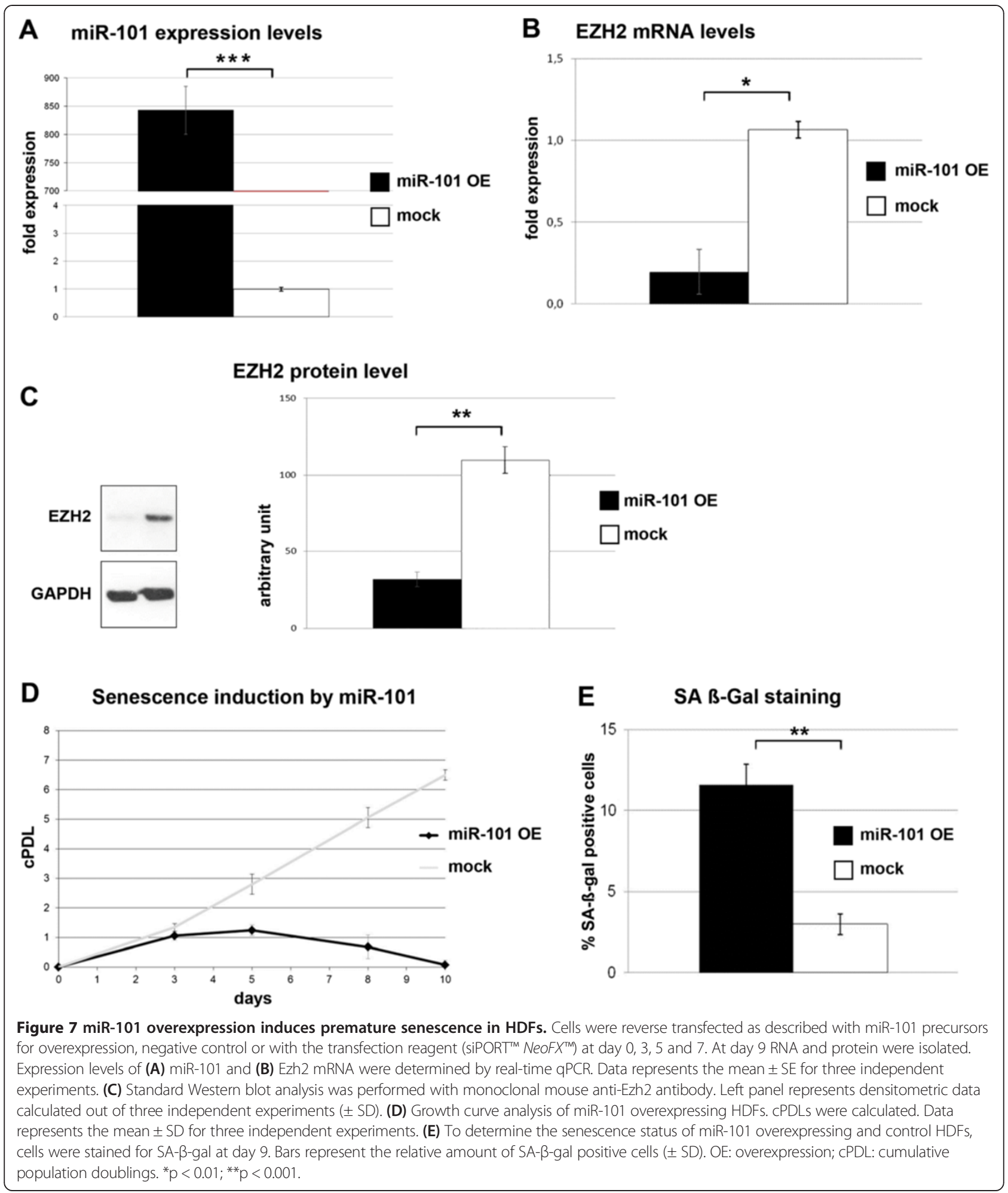

\section{UVB treatment}

For UVB treatment fibroblasts were seeded out in $10 \mathrm{~cm}$ dishes (Greiner Bio One, Austria) at a density of $6 \times 10^{5}$ (UVB) and $3 \times 10^{5}$ (control). Cells were washed with HBSS (Sigma) and covered with $2 \mathrm{ml}$ HBSS. To calculate the irradiation time, power per area $\left[\mathrm{W} / \mathrm{m}^{2}\right]$ was measured by a UVX radiometer (Thermo Fisher) and the following equation was used: irradiation time $[\mathrm{s}]=\mathrm{en}$ ergy per area $\left[\mathrm{J} / \mathrm{m}^{2}\right]$ divided by power per area $\left[\mathrm{W} / \mathrm{m}^{2}\right]$. The output of a Philips TL20W/01 lamp (Philips, The 
A miR-101 expression levels

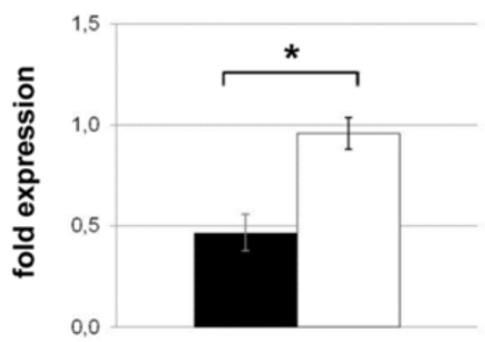

B

miR-101 expression levels

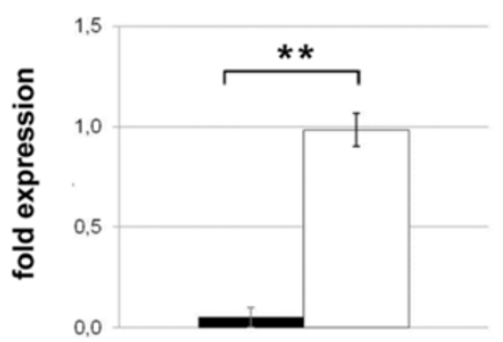

$\square \mathrm{miR}-101 \mathrm{KD}$

$\square$ control
EZH2 mRNA levels (control)

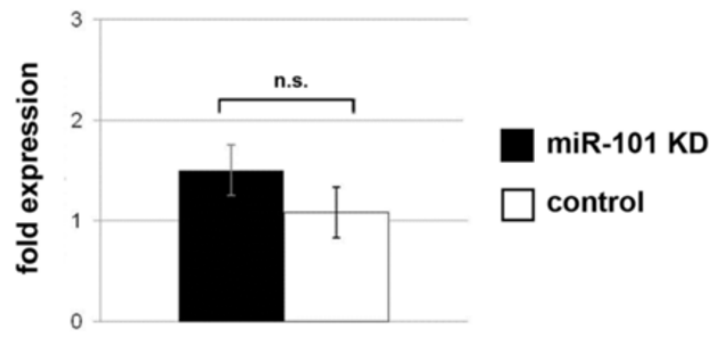

EZH2 mRNA levels (UVB)

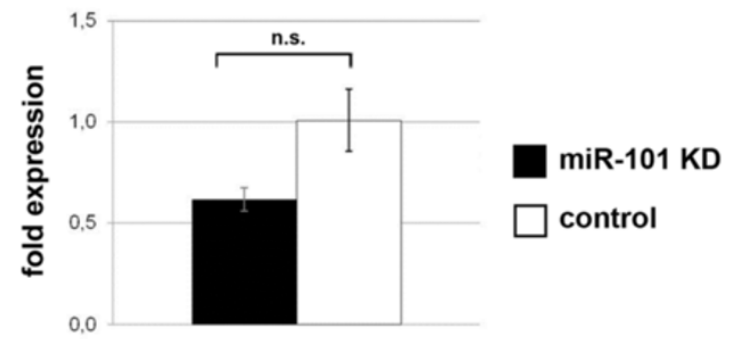

C

Control cells

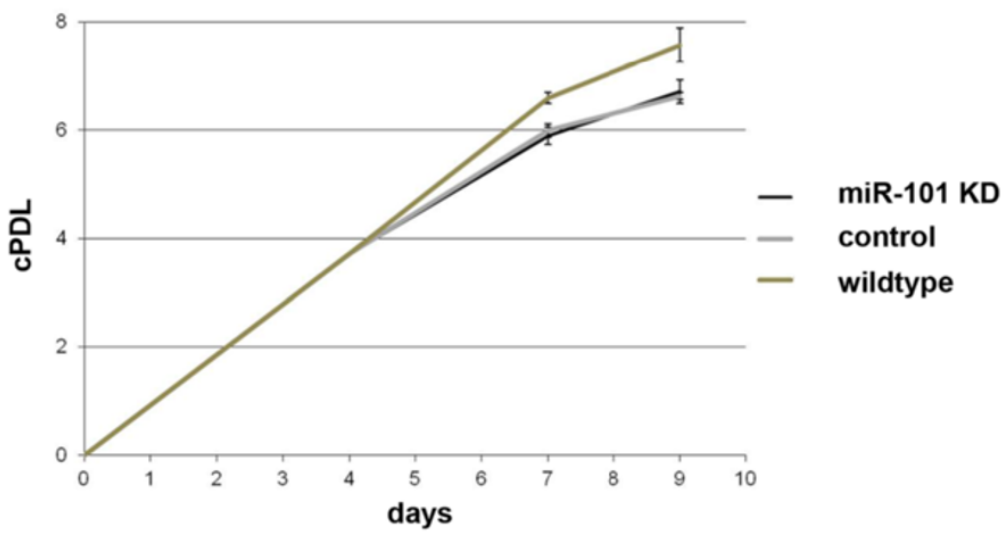

UVB treated cells

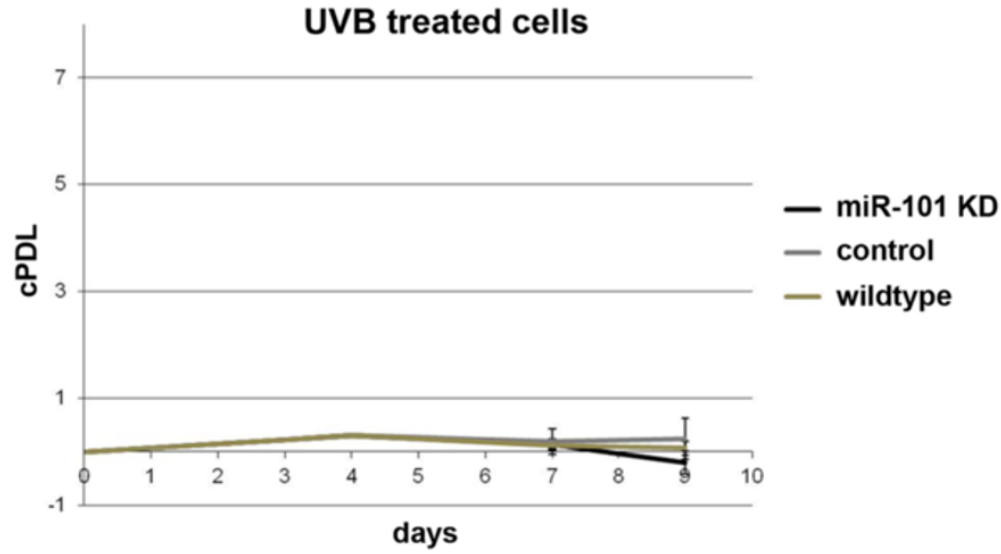

Figure $\mathbf{8}$ (See legend on next page.) 
(See figure on previous page.)

Figure 8 Depletion of miR-101 fails to prevent UVB-induced senescence. Cells were irradiated with UVB twice a day for 4 days. After the last exposure the cells were reverse transfected as described with miR-101 power inhibitors for knockdown, negative control or not treated. Reverse transfection was repeated at day 7. At day 9 RNA and protein were isolated. A. Control cells. Expression levels of miR-101 and Ezh2 mRNA of non-irradiated cells after miR-101 knockdown were determined by real-time GPCR. Bars indicate the mean \pm SE of three independent experiments. B. UVB-treated cells miR-101 and Ezh2 expression levels from UVB treated cells after miR-101 knockdown were determined by real-time qPCR. Bars indicate the mean \pm SE of three independent experiments. C. Growth curve analysis of miR-101 knockdown cells. CPDLs were calculated as described. Data represent the mean \pm SD of three independent experiments. CPDL: cumulative population doublings KD: $k$ nockdown. ${ }^{*} p<0.01$; ${ }^{* *} p<0.001$.

Netherlands) for $10 \mathrm{~cm}$ dishes was determined as $14.2 \pm$ $0.5 \mathrm{~W} / \mathrm{m}^{2}$. To test the sublethal dosage, cells were irradiated with $3000,3500,4000$ and $5000 \mathrm{~J} / \mathrm{m}^{2}$. For experiments, cells were irradiated twice a day with a dose of $4000 \mathrm{~J} / \mathrm{m}^{2}$ for 4 consecutive days, where irradiation time was $282 \pm 10 \mathrm{~s}$. UVB treatment of miRNA knockdown cells were performed in 6-well plates at a density of $1.3 \times 10^{5}$ cells (UVB) and $5 \times 10^{4}$ cells (control). The output of a Philips TL20W/01 lamp for 6-well plates was determined as $17.3 \pm 0.5 \mathrm{~W} / \mathrm{m}^{2}$ and irradiation time was $231 \pm 6 \mathrm{~s}$. After the first (day 1) and the last (day 4) irradiation, as well as after day 7 , day 9 and day 15 after the first irradiation, cells were lysed and RNA and protein were isolated.

\section{Protein isolation}

For the preparation of whole cell lysates, HDFs were washed twice with cold PBS and scraped off on ice in lysis buffer $(50 \mathrm{mM}$ Tris- $\mathrm{HCl}, 150 \mathrm{mM} \mathrm{NaCl}, 1 \% \mathrm{NP}-$ 40, 0.25\% Na-deoxycholate, $1 \mathrm{mM}$ EDTA, $100 \mathrm{nM}$ $\mathrm{Na}_{3} \mathrm{VO}_{4}, 1 \mathrm{mM} \mathrm{NaF}, 10 \mathrm{mM} \quad \beta$-glycerophosphate, $\mathrm{pH}$ 7.4) from the 6-well plate or $10 \mathrm{~cm}$ dish with a rubber policeman. Cells were three times deep-frozen in liquid nitrogen and thawed and further kept on ice for 30 minutes. After centrifugation at $20,000 \times g$ for $10 \mathrm{mi}-$ nutes at $4^{\circ} \mathrm{C}$, supernatant was used to determine the protein concentration by DC Protein Assay Kit (Biorad, Austria).

\section{mRNA and miRNA isolation}

Total RNA was isolated using either TRIzol $^{\circledR}$ Reagent (Invitrogen; for miRNA) or the RNeasy ${ }^{\circledR}$ Mini Kit (Qiagen; RNA) according the manufacturer's protocol. For the isolation of RNA with TRIzol ${ }^{\circledR}$ Reagent cells were trysinized, centrifuged, washed with $\mathrm{PBS}$ and resuspended in $1 \mathrm{ml}$ TRIzol ${ }^{\circledR}$ Reagent. After 5 minutes incubation at room temperature $200 \mu$ of Chloroform was added and vigorously vortexed. After 3 minutes incubation at room temperature a centrifugation step was performed for 10 minutes at $14000 \times g$ at $4^{\circ} \mathrm{C}$. The aqueous phase was transferred to a micro-centrifuge tube and mixed with $500 \mu \mathrm{l}$ isopropanol and incubated at room temperature for 10 minutes to precipitate the RNA. After 10 minutes centrifugation at $14000 \times g\left(4^{\circ} \mathrm{C}\right)$ the supernatant was discarded and the pellet was washed with ethanol (70\%) and centrifuged again for 5 minutes at $6000 \times g\left(4^{\circ} \mathrm{C}\right)$. The supernatant was discarded, the pellet air dried and re-suspended in $30 \mu \mathrm{l}$ of RNAse free water. Using the RNeasy ${ }^{\circledR}$ Mini Kit up to $5 \times 10^{6}$ cells were trypsinized and lysed in $350 \mu \mathrm{l}$ RLT buffer (including $10 \mu \mathrm{l} \beta$ mercaptoethanol per $\mathrm{ml}$ ). The lysate was homogenously mixed by pipetting up and down a few times. One volume of $70 \%$ ethanol was added, mixed by pipetting and transferred to RNeasy spin column. After a centrifugation step at $12900 \times g$ for 30 seconds at room temperature, three washing steps were performed. First $700 \mu \mathrm{l}$ RW1 buffer, two times $500 \mu \mathrm{l}$ RPE (one volume RPE added to four volumes ethanol) whereas the last washing step was carried out for 2 minutes at maximum speed to dry the RNAbinding membrane. RNA was eluted using $30 \mu \mathrm{l}$ of RNAse-free water. RNA concentration was quantified by photometric measurement at $260 \mathrm{~nm}$ and $280 \mathrm{~nm}$.

\section{Immunoblotting}

Equal amounts of protein were subjected to SDS gel electrophoresis (10-12.5\% SDS/polyacrylamide gel) and transferred to PVDF membrane by wet electro-blotting $(300 \mathrm{~mA}, 1 \mathrm{~h})$ using the standard Western blot protocol. The membranes were blocked with $5 \%$ skim milk in PBS-T (Phosphate buffered saline $+0.1 \%$ Tween 20) or in 5\% BSA in PBS-T for the detection of phosphorylated proteins for $1 \mathrm{~h}$ at room temperature. Primary antibodies were incubated for $1 \mathrm{~h}$ at room temperature or overnight at $4{ }^{\circ} \mathrm{C}$. After two times of washing with PBS$\mathrm{T}$, the second antibody was incubated for $45 \mathrm{~min}$ at room temperature. After a few washing steps with PBS$\mathrm{T}$, immune-reactive proteins were detected using an enhanced chemiluminescence system (ECL+, Amersham Life Science, Germany). The following antibodies were used: mouse monoclonal anti-p21 WAF1 (Pharmingen, \#556430) mouse monoclonal anti-pRb (Pharmingen, \#554136), mouse monoclonal anti-p53 (Santa Cruz, \#sc126), rabbit polyclonal anti-GAPDH (Santa Cruz, \#sc25778), rabbit polyclonal phospho-p53 (Ser15; Cell Signaling, \#9284), mouse monoclonal $\alpha$-Tubulin (Sigma, \#t-5168), mouse monoclonal ant-Cyclin D1 (Neomarkers, \#MS-210-P) and mouse monoclonal anti-Ezh2 (BD Biosciences, \#612666), mouse monoclonal anti-p16 ${ }^{\mathrm{INK} 4 \mathrm{~A}}$ 
(BD Biosciences, \#511325GR), rabbit polyclonal antip15 ${ }^{\text {INK4b }}$ (Abcam, \#ab53034), mouse monoclonal antiRUNX1/AML1 (Abcam, \#ab54869), rabbit monoclonal anti-retinoic acid receptor beta - RARB (abcam, \#ab124701). As secondary antibodies, polyclonal antibodies from Dako were used. For positive control, U2-OS cells were transiently transfected with a pcDNA3.1 vector carrying the sequence of either Ezh2 or p16 ${ }^{\mathrm{INK} 4 \mathrm{a}}$.

\section{Real-time q-PCR analysis of mRNAs}

RNA was isolated using the RNeasy ${ }^{\circledR}$ Mini Kit (Qiagen) and quantified as described above. For cDNA synthesis 0.4-1 $\mu \mathrm{g}$ of total RNA was reverse transcribed with the Transcriptor First Strand cDNA Synthesis Kit purchased from Roche Applied Science and diluted 1:4. Amplification of Ezh2 mRNA was carried out with the following primers: (forward) 5'-CAT TCG GTA AAT CCA AAC TGC-3' and (reverse) 5'-CGA CAT ACT TCA GGG CAT CA-3'. RUNX1 (Runt-related transcription factor 1) (forward) 5'-CCC TCG TGC CTC CCT GAA CCA-3' ; (reverse) 5'-GGC TGG GGA GAG GGA TGG ACA-3'. VEGFA (Vascular endothelial growth factor A) (forward) 5'TTT GCT TGC CAT TCC CCA C-3'; (reverse) 5'GCT CTT GCT ACC TCT TTC CTC-3'. CDKN2B (Cyclin-dependent kinase 4 inhibitor B) (forward) 5'GAT GAG GAC AAT GAG GCA AAG-3' ; (reverse) 5'TGG GAA GAA AAG CAA GAC AAC-3'. NOTCH3 (Neurogenic locus notch homolog protein 3) (forward) 5'-TCA TCC TCT TCT CTT TCC ACC-3'; (reverse) 5'TCC CAG ACT CTT CAC AAG AC-3'. As a housekeeper B2M ( $\beta-2$ microglobulin) was amplified with the following primers: (forward) 5'-GAA TTC ACC CCC ACT GAA AA-3' and (reverse) 5'-CTC CAT GAT GCT GCT TAC A-3' and GAPDH (Glyceraldehyde 3-phosphate dehydrogenase) (forward) 5'-GAG TCA ACG GAT TTG GTC GT-3' ; (reverse) 5'-GAT CTC GCT CCT GGA AGA TG-3'. Real-time q-PCR was performed in duplicates using the SYBR Green I Master Mix in the LightCycler ${ }^{\circledR} 480$ Instrument (Roche Applied Science).

\section{Real-time q-PCR analysis of microRNAs}

RNA was extracted with TRIzol ${ }^{\circledR}$ Reagent (Invitrogen, as described above). Using the Universal cDNA Synthesis Kit (Exiqon, Denmark) cDNA synthesis was performed according the manufacturer's protocol. To perform realtime PCR SYBR Green master mix and specific primer for miR-101 (hsa-miR-101, LNA PCR primer set, UniRT; Exiqon, Denmark) were used. Expression levels of miR101 were normalized to endogenous 5S rRNA (Exiqon, Denmark). In addition, 7 other microRNAs (hsa-miR155, hsa-miR-15a, hsa-miR-17, hsa-miR-20a, hsa-miR20b, hsa-miR-34a, hsa-miR-93) were chosen for qPCR confirmation of array data using the Taqman qPCR platform (Life Technologies). In brief, specific reverse transcription (RT) reactions were performed with each microRNA primer and RNU44 as endogenous control using $10 \mathrm{ng}$ of total RNA as input material. Following $\mathrm{RT}$, qPCRs were run in 4 replicates using the Taqman Universal Mastermix (Life Technologies) on a Rotor-Gene Q (Qiagen) according to the manufacturer's protocol. Data analysis was performed using the ddCt method.

\section{Genome-wide RNA profiling (microarray)}

Total RNA was isolated using the RNeasy ${ }^{\circledR}$ Mini Kit (Qiagen, as described above). After quantification RNA was sent to The Microarray Facility (Tübingen, Germany). The obtained data set was analyzed using CARMAweb 1.5 (https://carmaweb.genome.tugraz.at/carma/). A cut-off of 1.5 fold change was used to determine the total number of up- and downregulated genes.

\section{Low density array (TaqMan q-PCR)}

RT-q-PCR was performed using Taqman ${ }^{\circledR}$ Low Density Array technology (Applied Biosystems). A total of 93 candidate and three housekeeper genes were used to design Taqman ${ }^{\circledR}$ custom array. According to the manufacturer's protocol q-PCR was performed, as described [60].

\section{Locked nucleic acid (LNA)-miRNA microarray}

For miRNA expression profiling, LNA-miRNA microarrays (Exiqon, Denmark) consisting of 559 human and 170 mouse LNA-modified probes [61] against miRNAs annotated in Sanger miRBase v9.2 [62] as well as 77 not yet annotated probes (miRPlus, Exiqon, Denmark) were used as previously described [16]. In brief, total RNA extracts were end-labeled using $\mathrm{Cy} 3$ dye and hybridized against a common reference RNA-pool end-labeled with Cy5 on a Tecan HS 400 hybridization station (Tecan, Austria). Arrays were scanned at $10 \mu \mathrm{M}$ resolution (Axon Genepix 4000B, Axon Instruments) and raw intensities were extracted using GenePixPro 4.1 software (Axon Instruments). The acquired array signal data was further processed under R/Bioconductor using linear models for microarray analysis [63] and differential expression between control and UVB treated cells was calculated using t-statistics and p-value adjustment to multiple testing according to Benjamini-Hochberg.

\section{Stable overexpression and knockdown of Ezh2}

For the overexpression of Ezh2 the lentiviral pLenti6/ V5-DEST Gateway vector (Invitrogen) was used. Cloning included the TOPO cloning of Ezh2 into pENTR/DTOPO. This vector was used to introduce the Ezh2 coding sequence into pLenti6/V5-DEST by recombination to generate the transfer vector pLenti6-Ezh2 (for further details see Invitrogen's ViraPower Lentiviral Expression System manual). The GFP-control vector was generated in the same way. As a transfer vector for knockdown of 
Ezh2 lentiviral pLKO.1-TRC short-hairpin vector were purchased from Addgene/Open Biosystems (United Kindom). The following sequences were chosen: pLKO73 within the 3'UTR of Ezh2 (5'-TAT TGC CTT CTC ACC AGC TGC-3') and pLKO-75 within the coding region (5'-CCA ACA CAA GTC ATC CCA TTA-3'). As a control the empty vector was used. For packaging of the lentivirus in HEK293FT cells (Invitrogen), $3 \mu \mathrm{g}$ of the corresponding overexpression or knockdown vector was combined with $7.5 \mu \mathrm{g}$ vector backbone psPAX2, $2.5 \mu \mathrm{g}$ envelope encoding plasmid pMD2.G. HEK293FT cells were cultivated in T75 flasks to $90 \%$ confluence and transfected with the mixture using Lipofectamine 2000 (Invitrogen). The next day medium was replaced by $10 \mathrm{ml}$ of growth medium without antibiotics. After 48 hours (day 5) the supernatant was harvested, centrifuged at $300 \times \mathrm{g}$ for 5 minutes at room temperature and filtered through a $0.45 \mu \mathrm{m}$ PVDF filter (Millipore, Vienna, Austria). Afterwards, the virus containing supernatant was concentrated using Polyethylene glycol. Therefore, to one volume Polyethylene glycol solution (50 mM Polyethylene glycol, $41 \mathrm{mM} \mathrm{NaCl}$, autoclave, $\mathrm{pH}=7.2$; PEG) four volumes of supernatant was added and incubated for 2 hours at $4^{\circ} \mathrm{C}$, carefully mixing every 20-30 minutes by inverting. The solution was centrifuged at $1500 \times \mathrm{g}$ for 30 minutes at $4^{\circ} \mathrm{C}$ and a white pellet should be visible. After aspirating the supernatant an additional centrifugation step was carried out at $1500 \times \mathrm{g}$ for 5 minutes at $4^{\circ} \mathrm{C}$ to collect remaining PEG which is aspirated carefully. The white pellet was re-suspended in medium by pipetting up and down and vigorously vortexing for 20 to 30 seconds. As a guideline $500 \mu \mathrm{l}$ for one T75 flask was used, aliquoted to $100 \mu \mathrm{l}$ and stored at $-80^{\circ} \mathrm{C}$. The titer of the concentrated lentiviral supernatant was determined by seeding $5 \times 10^{4}$ $\mathrm{U} 2-\mathrm{OS}$ in 6 -well plates with $8 \mathrm{mg} / \mathrm{mL}$ hexadimethrine bromide (polybrene; Sigma-Aldrich) as transduction enhancer. A stock of $4 \mathrm{mg} / \mathrm{mL}$ of polybrene was prepared by resolving polybrene in sterile water and filtered through a $0.22 \mu \mathrm{m}$ sterile filter. The following day the medium was replaced by $2 \mathrm{ml}$ of DMEM. After day 3 the selection was started. For overexpression selection $10 \mu \mathrm{g} / \mathrm{ml}$ Blasticidin, for knockdown $500 \mathrm{ng} / \mathrm{ml}$ puromycin was added to each well. The antibiotic containing medium was replaced every second day. Approximately 6-7 days after starting the selection the untransduced cells are dead. For the staining the cells are washed three times with PBS, covered with crystal violet and incubated for 5-10 minutes at room temperature. Wells are washed twice with ddH2O and air dried. The colonies are counted and multiplied with 1000 and the corresponding dilution. This procedure allows to calculate transfection units (TU) of the virus/ml.

\section{Overexpression and knockdown of miRNAs}

To achieve the overexpression of microRNA in HDFs, cells were reverse transfected with Pre-miR ${ }^{\mathrm{Tm}}$ miRNA Precursor for miR-15a, miR-20a, miR-93, miR-101, and Pre-miR ${ }^{\mathrm{TM}}$ miRNA Precursor Molecules-Negative Control \#2 for negative control (Applied Biosystems, Austria) using siPORT $^{\mathrm{TM}} \mathrm{NeoFX}^{\mathrm{TM}}$ Transfection Agent (Ambion, Austria) according the manufacturer's protocol. Cells were trysinized, counted and $6 \times 10^{4}$ cells were centrifuged to be resuspended in $1.8 \mathrm{ml}$ medium. The transfection reagent siPORT $^{\mathrm{Tm}} \mathrm{NeoFX}^{\mathrm{sm}}$ and Opti-MEM medium (Invitrogen) were adjusted to room temperature. Opti-MEM medium was mixed with Precursors and with the transfection reagent and incubated for 10 minutes incubation at room temperature. Both reagents are mixed and additionally incubated at room temperature for 10 minutes. Afterwards, the mixture is added together with the cell suspension to 6-well plates [22]. Cells were UVB treated as described above. Experiments were done in 6-well plates with $1.3 \times 10^{5}$ (UVB) and $5 \times 10^{4}$. After the last exposure, cells were trypsinized and prepared for transfection. For miR-101 knockdown, cells were reverse transfected with miRCURY LNA ${ }^{\mathrm{mm}}$ microRNA Power Inhibitor and miRCURY LNA ${ }^{\mathrm{Tm}}$ microRNA Power Inhibitor Negative Control A for negative control (Exiqon, Denmark) using siPORT ${ }^{\mathrm{Tm}}$ NeoFX $^{\mathrm{TM}}$ Transfection Agent (Ambion, Austria) according the manufacturer's protocol. UVB treated cells were trypsinized, counted, centrifuged and re-suspended in $1.8 \mathrm{ml}$ medium. Opti-MEM medium was mixed with the miRNA inhibitor or control and with the transfection reagent and incubated for $10 \mathrm{mi}$ nutes at room temperature. The protocol was continued as described above. For both, Power Inhibitor and Negative Control A 15 nM (day 4 and day 7) were used.

\section{Transduction of cells by lentiviral particles}

For transduction $5 \times 10^{4}$ human diploid fibroblasts (HDFs) are seeded to 6-well plates the day before (day 1). A multiplicity of infection of two together with $8 \mu \mathrm{g} / \mathrm{ml}$ polybrene as transduction enhancer was used in a total of one milliliter. The medium was changed the next day and selection was started at day 3. For the selection of Ezh2 overexpressing cells $10 \mu \mathrm{g} / \mathrm{ml}$ blasticidin, for Ezh2 knockdown cells $500 \mathrm{ng} / \mathrm{ml}$ puromycin was used.

Bioinformatics analysis for predicting miRNA target genes For miRNA target prediction the following tools were used: TargetScan [64], PicTar [65], miRanda [66], PITA [67], ElMMo [68], RNA22 [69], DIANA-microT [70] and GenMiR++[71]. The predictions of these tools reflect miRNA:mRNA pairing, site location, conservation, site accessibility, multiple sites and expression profile [72]. The results were visualized using in house developed tools 
(Genesis [73] and ClueGO [74]) as well as publicly available tools: Cytoscape [75].

\section{Statistical analysis}

We used limma package [63] for miRNA microarray analysis. T-test and adjusted p-value were used to identify genes differentially expressed between control and UVB treated cells. Furthermore, we also investigated the linear relationship between miRNAs and their high confidence target genes by using Pearson correlation coefficients. All analyses were done with the use of statistical software programs R/Bioconductor.

\section{Pathway analysis and network visualization}

The data set obtained from the genome-wide RNA profiling was uploaded to Ingenuity Systems (http://www. ingenuity.com/) and pathway analysis was done. The visualization of the network was done using the opensource Cytoscape software platform [75] for visualizing biomolecular interaction networks. The color and shape of nodes are based on different node attributes available for the analyzed dataset. The red triangles, purple circles and orange diamonds in the network are indicating miRNAs, target genes, and transcription factors, respectively. Edges represent correlation between miRNAs and mRNAs, the color of the edges designate the type of interaction. Red is for positive and green is for negative correlation. The biological functions triggered by the miRNA through its target genes were visualized using ClueGO [74] a Cytoscape plugin.

\section{Additional files}

Additional file 1: Figure S1. Growth characteristics of UVB treated cells. Human diploid fibroblasts (HDF) were UVB treated in $10 \mathrm{~cm}$ dishes with $4000 \mathrm{~J} / \mathrm{m}^{2}$ as described. (A) Cumulative population doubling (CPDL) of UVB treated and untreated control cells were calculated at the indicated time points as described in material and methods. (B) To determine the senescence status of HDFs, cells were stained for SA- $\beta$-gal at day 9 after the first irradiation. Bars indicate the relative percentage of SA- $\beta$-gal-positive cells ( $\pm S D)$; results were derived from three independent experiments. (C) Protein was isolated from UVB irradiated and control cells at the indicated time points. Defined protein levels were determined by standard Western blot analysis. Experiments were performed in duplicates. As were used, as indicated. As positive control for the right panel, lysates from mock-transfected (U2-OS pX) and p16INK4A transfected (U2-OS p16) U2-OS osteosarcoma cells were used, as indicated. CPDL: cumulative population doublings; $\mathrm{Co}$ : control.

Additional file 2: Figure S2. Senescence status of HFF1 and PFF. HFF1 and PFF were UVB irradiated in 6-well lates with 3000, 3500, 4000, 4500 and $5000 \mathrm{~J} / \mathrm{m}^{2}$ as described. (A) Cumulative population doublings (CPDL) of UVB treated and untreated cells were calculated at the indicated time points as described in material and methods. (B) Cells were stained for $S A-\beta$-gal at $d 9$ after the first irradiation to determine the senescence status. Bars indicate the relative percentage of $\beta$-gal-positive cells $( \pm S D)$; results were derived from three independent experiments.

Additional file 3: Figure S3. Protein levels of UVB irradiated HDF. Protein was isolated from UVB irradiated and control cells at the indicated time points. Protein levels were analyzed by standard Western blot. Experiments were performed in triplicates. In the lower panels, controls for the Western blots are provided as follows: Left panel: p-p53 Western blot in untreated vs. cisplatin-treated HFF-1. Middle panel: Ezh2 Western blot in U-2OS cells transfected with pcDNA3-Ezh2 or empt vector (EV), as indicated. Right panel: p16INK4A Western blot iin U-2OS cells transfected with pcDNA3-p16INK4A or empty vector (EV), as indicated.

Additional file 4: Table S1. Genome-wide transcriptome analysis after UVB treatment. RNA was isolated from UVB treated and control cells. Genome-wide RNA profiling (microarray) was performed at different time points. A cutoff of $>1.5$ fold regulation was used for the result of day 7 and day 9 after UVB irradiation. ${ }^{*}$ indicates significant changes in gene expression at day 9 , derived from three independent Affymetrix chip experiments.

Additional file 5: Table S2. microRNA expression levels after UVB treatement. miRNA was isolated from UVB treated and control cells. miRNA expression levels were determined by Locked nucleic acid (LNA)-miRNA microarray. A cut-off of $>1.5$ fold regulation was used. Adjusted p-values of $\leq 0.05$ are in black colour.

Additional file 6: Figure S4. Validation of selected miRNA regulatory interactions. miR-15a and miR-20a were overexpressed in HDF as indicated. Extracts were prepared from cells overexpressing the indicated miRNAs and probed with antibodies to RUNX1, CDKN2B, and RARB, as indicated.

Additional file 7: Figure S5. Depletion of Ezh2 induces premature senescence in HDFs. Knockdown of Ezh2 was done with two different constructs in HDFs as described. (A) CPDLs were calculated for Ezh2 knockdown cells and scrambled transfected cells. Data represents the mean \pm SD of three independent experiments. Representative standard western blot analysis of Ezh2 knockdown. (B) SA- $\beta$-gal activity was determined at day 9 after transfection. Bars represent the mean \pm SD of three independent experiments. CPDL: cumulative population doublings; scr: scrambled shRNA; shRNA: small hairpin RNA.

Additional file 8: Figure S6. Ezh2 protein and mRNA levels after lentiviral Ezh2 overexpression. HDFs were transfected with Ezh2 or GFP overexpression virus. As an additional control HDF wildtype were used. RNA and protein were isolated as described. For quantification of Ezh2 mRNA levels real-time qPCR was performed (left panel). Protein lysates were subjected to SDS-page and analyzed for Ezh2 protein levels by standard Western Blot. Number in brackets represent densitometric data.

\section{Abbreviations}

ECL: Enhanced chemoluminescence; Ezh2: Enhancer of zeste homolog 2; HBSS: Hanks' balanced salt slution; HDF: Human diploid fibroblasts; MAPK: Mitogen-activated protein kinase; miR: microRNA; PBS: Phosphatebuffered saline; pRb: Retinoblastoma protein; PDL: Population doublings; RT-QPCR: Real-time quantitative PCR; SA-ß-gal: Senescence-associated B-galactosidase; shRNA: Small hairpin RNA; UVB: Ultraviolet light band B.

\section{Competing interests}

The authors declared that they have no competing interests.

\section{Authors' contributions}

PJD, ZT and JG designed the research. EH performed the transcription factor binding sites analysis. MH, RM and MS did the Locked nucleic acid (LNA)miRNA microarrays for determination of microRNA levels. UVB irradiation experiments and collection of samples were done with the help of MN by $R G$. RG, $A P$, and $M C$ performed microRNA knockdown and overexpression experiments, determination of protein levels and bioinformatics. PC analyzed data using bioinformatics tools. Cloning of the Ezh2 vector and the calculation of expression levels of genes of the genome-wide RNA profiling and low density arrays were done with the help of CM, MN and LM. PJD and RG wrote the manuscript. All the authors read and approved the final manuscript.

\section{Acknowledgements}

We acknowledge excellent technical support by Michael Neuhaus and Hans-Peter Viertler. The work was supported by grants from the Austrian Science Funds (NFN S93 and SFB021). Genome Research Austria GEN-AU (Project 820982 "Non-coding RNAs" and "Bioinformatics Integration Network"); and 
grants by the Herzfelder'sche Familienstiftung and CE.R.I.E.S to JG, and a BOKU Doc scholarship to $\mathrm{MH}$, and Lise-Meitner Scholarship to RM.

\section{Author details}

'Institute for Biomedical Aging Research, Austrian Academy of Sciences, Rennweg 10, Innsbruck 6020, Austria. ${ }^{2}$ Department of Biotechnology, BOKU University of Natural Resources and Life Sciences Vienna, Muthgasse 18, Vienna 1190, Austria. ${ }^{3}$ Division for Bioinformatics, Biocenter, Medical University Innsbruck, Innrain 52, Innsbruck 6020, Austria. ${ }^{4}$ Institute for Genomics and Bioinformatics, University of Technology, Petersgasse 14, Graz 8010, Austria. ${ }^{5}$ Evercyte GmbH, Muthgasse 18, Vienna, A 1190, Austria.

Received: 29 March 2012 Accepted: 8 March 2013

Published: 4 April 2013

\section{References}

1. Vigneron A, Vousden $\mathrm{KH}: \mathrm{p} 53, \mathrm{ROS}$ and senescence in the control of aging. Aging (Albany NY) 2010, 2(8):471-474

2. Thomas DM, Yang HS, Alexander K, Hinds PW: Role of the retinoblastoma protein in differentiation and senescence. Cancer Biol Ther 2003, 2(2):124-130.

3. Lukas J, Parry D, Aagaard L, Mann DJ, Bartkova J, Strauss M, Peters G, Bartek J: Retinoblastoma-protein-dependent cell-cycle inhibition by the tumour suppressor p16. Nature 1995, 375(6531):503-506.

4. Ressler S, Bartkova J, Niederegger H, Bartek J, Scharffetter-Kochanek K, Jansen-Durr $\mathrm{P}$, Wlaschek M: p16INK4A is a robust in vivo biomarker of cellular aging in human skin. Aging Cell 2006, 5(5):379-389.

5. Narita M, Nunez S, Heard E, Lin AW, Hearn SA, Spector DL, Hannon GJ, Lowe SW: Rb-mediated heterochromatin formation and silencing of E2F target genes during cellular senescence. Cell 2003, 113(6):703-716.

6. Beausejour CM, Krtolica A, Galimi F, Narita M, Lowe SW, Yaswen P, Campisi J: Reversal of human cellular senescence: roles of the p53 and p16 pathways. EMBO J 2003, 22(16):4212-4222.

7. Passos JF, Nelson G, Wang C, Richter T, Simillion C, Proctor CJ, Miwa S, Olijslagers S, Hallinan J, Wipat A, et al: Feedback between p21 and reactive oxygen production is necessary for cell senescence. Mol Syst Biol 2010, 6:347.

8. Campisi J: Cellular senescence: putting the paradoxes in perspective. Curr Opin Genet Dev 2011, 21(1):107-112.

9. Toussaint O, Remacle J, Dierick JF, Pascal T, Frippiat C, Zdanov S, Magalhaes $J$, Royer V, Chainiaux F: From the hayflick mosaic to the mosaics of ageing. Role of stress-induced premature senescence in human ageing. Int J Biochem Cell Biol 2002, 34(11):1415-1429.

10. Chainiaux F, Magalhaes JP, Eliaers F, Remacle J, Toussaint O: UVB-induced premature senescence of human diploid skin fibroblasts. Int J Biochem Cell Biol 2002, 34(11):1331-1339.

11. Borlon C, Vankoningsloo S, Godard P, Debacq-Chainiaux F, Toussaint O: Identification of p53-dependent genes potentially involved in UVBmediated premature senescence of human skin fibroblasts using siRNA technology. Mech Ageing Dev 2008, 129(3):109-119.

12. Takashima A: UVB-dependent modulation of epidermal cytokine network: roles in UVB-induced depletion of langerhans cells and dendritic epidermal T cells. J Dermatol 1995, 22(11):876-887.

13. el-Ghorr AA, Norval M: Biological effects of narrow-band (311 nm TL01) UVB irradiation: a review. J Photochem Photobiol B 1997, 38(2-3):99-106.

14. Almeida MI, Reis RM, Calin GA: MicroRNA history: discovery, recent applications, and next frontiers. Mutat Res 2011, 717(1-2):1-8.

15. Thomas M, Lieberman J, Lal A: Desperately seeking microRNA targets. Nat Struct Mol Biol 2010, 17(10):1169-1174.

16. Hackl M, Brunner S, Fortschegger K, Schreiner C, Micutkova L, Muck C, Laschober GT, Lepperdinger G, Sampson N, Berger $P$, et al: miR-17, miR19b, miR-20a, and miR-106a are down-regulated in human aging. Aging Cell 2010, 9(2):291-296.

17. Liang R, Bates DJ, Wang E: Epigenetic control of MicroRNA expression and aging. Curr Genomics 2009, 10(3):184-193.

18. Grillari J, Grillari-Voglauer R: Novel modulators of senescence, aging, and longevity: Small non-coding RNAs enter the stage. Exp Gerontol 2010, 45(4):302-311

19. Chen W, Kang J, Xia J, Li Y, Yang B, Chen B, Sun W, Song X, Xiang W, Wang $X$, et al: p53-related apoptosis resistance and tumor suppression activity in UVB-induced premature senescent human skin fibroblasts. Int J Mol Med 2008, 21(5):645-653.

20. Dumaz N, Drougard C, Quilliet X, Mezzina M, Sarasin A, Daya-Grosjean L: Recovery of the normal $\mathrm{p} 53$ response after UV treatment in DNA repair-deficient fibroblasts by retroviral-mediated correction with the XPD gene. Carcinogenesis 1998, 19(9):1701-1704.

21. Di Leonardo A, Linke SP, Clarkin K, Wahl GM: DNA damage triggers a prolonged p53-dependent G1 arrest and long-term induction of Cip1 in normal human fibroblasts. Genes Dev 1994, 8(21):2540-2551.

22. England NL, Cuthbert AP, Trott DA, Jezzard S, Nobori T, Carson DA, Newbold RF: Identification of human tumour suppressor genes by monochromosome transfer: rapid growth-arrest response mapped to $9 \mathrm{p} 21$ is mediated solely by the cyclin-D-dependent kinase inhibitor gene, CDKN2A (p16INK4A). Carcinogenesis 1996, 17(8):1567-1575.

23. Ahmed NU, Ueda M, Ichihashi M: Induced expression of p16 and p21 proteins in UVB-irradiated human epidermis and cultured keratinocytes. J Dermatol Sci 1999, 19(3):175-181.

24. Medrano EE, Im S, Yang F, Abdel-Malek ZA: Ultraviolet B light induces G1 arrest in human melanocytes by prolonged inhibition of retinoblastoma protein phosphorylation associated with long-term expression of the p21Waf-1/SDI-1/Cip-1 protein. Cancer Res 1995, 55(18):4047-4052.

25. Fang $Y, H u X H$, Jia ZG, Xu MH, Guo ZY, Gao FH: Tiron protects against UVB-induced senescence-like characteristics in human dermal fibroblasts by the inhibition of superoxide anion production and glutathione depletion. Australas J Dermatol 2012, 53(3):172-180.

26. Tagawa H, Karube K, Tsuzuki S, Ohshima K, Seto M: Synergistic action of the microRNA-17 polycistron and Myc in aggressive cancer development. Cancer Sci 2007, 98(9):1482-1490.

27. Wang Z, Liu M, Zhu H, Zhang W, He S, Hu C, Quan L, Bai J, Xu N: Suppression of p21 by c-Myc through members of miR-17 family at the post-transcriptional level. Int J Oncol 2010, 37(5):1315-1321.

28. Biyashev D, Qin G: E2F and microRNA regulation of angiogenesis. Am J Cardiovasc Dis 2011, 1(2):110-118.

29. Long J, Wang Y, Wang W, Chang BH, Danesh FR: Identification of microRNA-93 as a novel regulator of vascular endothelial growth factor in hyperglycemic conditions. J Biol Chem 2010, 285(30):23457-23465.

30. Choi OR, Lim IK: Loss of p21(Sdi1) expression in senescent cells after DNA damage accompanied with increase of miR-93 expression and reduced p53 interaction with p21(Sdi1) gene promoter. Biochem Biophys Res Commun 2011, 407(2):406-411.

31. Fu X, Tian J, Zhang L, Chen Y, Hao Q: Involvement of microRNA-93, a new regulator of PTEN/Akt signaling pathway, in regulation of chemotherapeutic drug cisplatin chemosensitivity in ovarian cancer cells. FEBS Lett 2012, 586(9):1279-1286.

32. Klein U, Lia M, Crespo M, Siegel R, Shen Q, Mo T, Ambesi-Impiombato A, Califano A, Migliazza A, Bhagat G, et al: The DLEU2/miR-15a/16-1 cluster controls B cell proliferation and its deletion leads to chronic lymphocytic leukemia. Cancer Cell 2010, 17(1):28-40.

33. Ageilan RI, Calin GA: Croce CM: miR-15a and miR-16-1 in cancer: discovery, function and future perspectives. Cell Death Differ 2010, 17(2):215-220.

34. Cao R, Zhang Y: SUZ12 is required for both the histone methyltransferase activity and the silencing function of the EED-EZH2 complex. Mol Cell 2004, 15(1):57-67.

35. Carvalho J, van Grieken NC, Pereira PM, Sousa S, Tijssen M, Buffart TE, Diosdado B, Grabsch H, Santos MA, Meijer G, et al: Lack of microRNA-101 causes E-cadherin functional deregulation through EZH2 up-regulation in intestinal gastric cancer. J Pathol 2012, 228(1):31-44.

36. He L, He X, Lim LP, de Stanchina E, Xuan Z, Liang Y, Xue W, Zender L, Magnus J, Ridzon D, et al: A microRNA component of the p53 tumour suppressor network. Nature 2007, 447(7148):1130-1134.

37. Chekulaeva M, Filipowicz W: Mechanisms of miRNA-mediated posttranscriptional regulation in animal cells. Curr Opin Cell Biol 2009, 21(3):452-460.

38. Yan H, Wu J, Liu W, Zuo Y, Chen S, Zhang S, Zeng M, Huang W: MicroRNA20a overexpression inhibited proliferation and metastasis of pancreatic carcinoma cells. Hum Gene Ther 2010, 21(12):1723-1734.

39. Pin AL, Houle F, Guillonneau M, Paquet ER, Simard MJ: Huot J: miR-20a represses endothelial cell migration by targeting MKK3 and inhibiting p38 MAP kinase activation in response to VEGF. Angiogenesis 2012, 15(4):593-608

40. Liu S, Patel SH, Ginestier C, Ibarra I, Martin-Trevino R, Bai S, McDermott SP, Shang $L$, Ke J, Ou SJ, et al: MicroRNA93 regulates proliferation and 
differentiation of normal and malignant breast stem cells. PLOS Genet 2012, 8(6):e1002751.

41. Yu XF, Zou J, Bao ZJ: Dong J: miR-93 suppresses proliferation and colony formation of human colon cancer stem cells. World J Gastroenterol 2011, 17(42):4711-4717.

42. Fang L, Deng Z, Shatseva T, Yang J, Peng C, Du WW, Yee AJ, Ang LC, He C, Shan SW, et al: MicroRNA miR-93 promotes tumor growth and angiogenesis by targeting integrin-beta8. Oncogene 2011, 30(7):806-821.

43. Xu D, He XX, Chang Y, Sun SZ, Xu CR, Lin JS: Downregulation of MiR-93 expression reduces cell proliferation and clonogenicity of HepG2 cells. Hepatogastroenterology 2012, 59(120):2367-2373.

44. Ivanovska I, Ball AS, Diaz RL, Magnus JF, Kibukawa M, Schelter JM, Kobayashi SV, Lim L, Burchard J, Jackson AL, et al: MicroRNAs in the miR-106b family regulate $\mathrm{p} 21 / \mathrm{CDKN} 1 \mathrm{~A}$ and promote cell cycle progression. Mol Cell Biol 2008, 28(7):2167-2174

45. Wong P, Iwasaki M, Somervaille TC, Ficara F, Carico C, Arnold C, Chen CZ, Cleary ML: The miR-17-92 microRNA polycistron regulates MLL leukemia stem cell potential by modulating p21 expression. Cancer Res 2010, 70(9):3833-3842

46. Ciccia A, Elledge SJ: The DNA damage response: making it safe to play with knives. Mol Cell 2010, 40(2):179-204.

47. Finnerty JR, Wang WX, Hebert SS, Wilfred BR, Mao G, Nelson PT: The miR-15/107 group of microRNA genes: evolutionary biology, cellular functions, and roles in human diseases. J Mol Biol 2010, 402(3):491-509.

48. Musumeci M, Coppola V, Addario A, Patrizii M, Maugeri-Sacca M, Memeo L, Colarossi C, Francescangeli F, Biffoni M, Collura D, et al: Control of tumor and microenvironment cross-talk by miR-15a and miR-16 in prostate cancer. Oncogene 2011, 30(41):4231-4242.

49. Pedley J, Ablett EM, Pettit A, Meyer J, Dunn IS, Sturm RA, Parsons PG: Inhibition of retinoblastoma protein translation by UVB in human melanocytic cells and reduced cell cycle arrest following repeated irradiation. Oncogene 1996, 13(6):1335-1342.

50. Tong X, Van Dross RT, Abu-Yousif A, Morrison AR, Pelling JC: Apigenin prevents UVB-induced cyclooxygenase 2 expression: coupled mRNA stabilization and translational inhibition. Mol Cell Biol 2007, 27(1):283-296.

51. Varambally S, Cao Q, Mani RS, Shankar S, Wang X, Ateeq B, Laxman B, Cao $X$, Jing $X$, Ramnarayanan K, et al: Genomic loss of microRNA-101 leads to overexpression of histone methyltransferase EZH2 in cancer. Science 2008, 322(5908):1695-1699.

52. Cao P, Deng Z, Wan M, Huang W, Cramer SD, Xu J, Lei M, Sui G: MicroRNA101 negatively regulates Ezh2 and its expression is modulated by androgen receptor and HIF-1alpha/HIF-1beta. Mol Cancer 2010, 9:108.

53. Bracken AP, Kleine-Kohlbrecher D, Dietrich N, Pasini D, Gargiulo G, Beekman C, Theilgaard-Monch K, Minucci S, Porse BT, Marine JC, et al: The polycomb group proteins bind throughout the INK4A-ARF locus and are disassociated in senescent cells. Genes Dev 2007, 21(5):525-530.

54. Fan T, Jiang S, Chung N, Alikhan A, Ni C, Lee CC, Hornyak TJ: EZH2dependent suppression of a cellular senescence phenotype in melanoma cells by inhibition of p21/CDKN1A expression. Mol Cancer Res 2011, 9(4):418-429.

55. Tzatsos A, Paskaleva P, Lymperi S, Contino G, Stoykova S, Chen Z, Wong KK, Bardeesy N: A lysine (K)-specific demethylase 2B (KDM2B)-let-7-enhancer of zester homolog $2(\mathrm{EZH} 2)$ pathway regulates cell cycle progression and senescence in primary cells. J Biol Chem 2011, 286(38):33061-33069.

56. Tang X, Milyavsky M, Shats I, Erez N, Goldfinger N, Rotter V: Activated p53 suppresses the histone methyltransferase EZH2 gene. Oncogene 2004, 23(34):5759-5769.

57. Tarasov V, Jung P, Verdoodt B, Lodygin D, Epanchintsev A, Menssen A, Meister $\mathrm{G}$, Hermeking $\mathrm{H}$ : Differential regulation of microRNAs by $\mathrm{p} 53$ revealed by massively parallel sequencing: miR-34a is a p53 target that induces apoptosis and G1-arrest. Cell Cycle 2007, 6(13):1586-1593.

58. Hutter E, Renner K, Pfister G, Stockl P, Jansen-Durr P, Gnaiger E: Senescenceassociated changes in respiration and oxidative phosphorylation in primary human fibroblasts. Biochem J 2004, 380(Pt 3):919-928.

59. Dimri GP, Lee X, Basile G, Acosta M, Scott G, Roskelley C, Medrano EE, Linskens M, Rubelj I, Pereira-Smith O, et al: A biomarker that identifies senescent human cells in culture and in aging skin in vivo. Proc Natl Acad Sci U S A 1995, 92(20):9363-9367.

60. Laschober GT, Ruli D, Hofer E, Muck C, Carmona-Gutierrez D, Ring J, Hutter E, Ruckenstuhl C, Micutkova L, Brunauer R, et al: Identification of evolutionarily conserved genetic regulators of cellular aging. Aging Cell 2010, 9(6):1084-1097.

61. Castoldi M, Schmidt S, Benes V, Noerholm M, Kulozik AE, Hentze MW, Muckenthaler MU: A sensitive array for microRNA expression profiling (miChip) based on locked nucleic acids (LNA). RNA 2006, 12(5):913-920.

62. Griffiths-Jones S, Saini HK, van Dongen S, Enright AJ: miRBase: tools for microRNA genomics. Nucleic Acids Res 2008, 36(Database issue):D154-D158.

63. Smyth GK: Linear models and empirical bayes methods for assessing differential expression in microarray experiments. Stat Appl Genet Mol Biol 2004, 3:Article3.

64. Grimson A, Farh KK, Johnston WK, Garrett-Engele P, Lim LP, Bartel DP: MicroRNA targeting specificity in mammals: determinants beyond seed pairing. Mol Cell 2007, 27(1):91-105.

65. Krek A, Grun D, Poy MN, Wolf R, Rosenberg L, Epstein EJ, MacMenamin P, da Piedade I, Gunsalus KC, Stoffel M, et al: Combinatorial microRNA target predictions. Nat Genet 2005, 37(5):495-500.

66. Griffiths-Jones S, Grocock RJ, van Dongen S, Bateman A, Enright AJ: miRBase: microRNA sequences, targets and gene nomenclature. Nucleic Acids Res 2006, 34(Database issue):D140-D144.

67. Kertesz M, lovino N, Unnerstall U, Gaul U, Segal E: The role of site accessibility in microRNA target recognition. Nat Genet 2007, 39(10):1278-1284.

68. Gaidatzis D, van Nimwegen E, Hausser J, Zavolan M: Inference of miRNA targets using evolutionary conservation and pathway analysis. BMC Bioinforma 2007, 8:69.

69. Miranda KC, Huynh T, Tay Y, Ang YS, Tam WL, Thomson AM, Lim B, Rigoutsos I: A pattern-based method for the identification of MicroRNA binding sites and their corresponding heteroduplexes. Cell 2006, 126(6):1203-1217.

70. Maragkakis M, Reczko M, Simossis VA, Alexiou P, Papadopoulos GL, Dalamagas T, Giannopoulos G, Goumas G, Koukis E, Kourtis K, et al: DIANAmicroT web server: elucidating microRNA functions through target prediction. Nucleic Acids Res 2009, 37(Web Server issue):W273-W276.

71. Huang JC, Babak T, Corson TW, Chua G, Khan S, Gallie BL, Hughes TR, Blencowe BJ, Frey BJ, Morris QD: Using expression profiling data to identify human microRNA targets. Nat Methods 2007, 4(12):1045-1049.

72. Saito T, Saetrom P: MicroRNAs-targeting and target prediction. N Biotechnol 2010, 27(3):243-249.

73. Sturn A, Quackenbush J, Trajanoski Z: Genesis: cluster analysis of microarray data. Bioinformatics 2002, 18(1):207-208.

74. Bindea G, Mlecnik B, Hackl H, Charoentong P, Tosolini M, Kirilovsky A, Fridman WH, Pages F, Trajanoski Z, Galon J: ClueGO: a cytoscape plug-in to decipher functionally grouped gene ontology and pathway annotation networks. Bioinformatics 2009, 25(8):1091-1093.

75. Shannon P, Markiel A, Ozier O, Baliga NS, Wang JT, Ramage D, Amin N, Schwikowski B, Ideker T: Cytoscape: a software environment for integrated models of biomolecular interaction networks. Genome Res 2003, 13(11):2498-2504.

doi:10.1186/1471-2164-14-224

Cite this article as: Greussing et al:: Identification of microRNA-mRNA functional interactions in UVB-induced senescence of human diploid fibroblasts. BMC Genomics 2013 14:224.

\section{Submit your next manuscript to BioMed Central and take full advantage of:}

- Convenient online submission

- Thorough peer review

- No space constraints or color figure charges

- Immediate publication on acceptance

- Inclusion in PubMed, CAS, Scopus and Google Scholar

- Research which is freely available for redistribution 